\title{
Genome-wide analysis of major intrinsic proteins in the tree plant Populus trichocarpa: Characterization of XIP subfamily of aquaporins from evolutionary perspective
}

\author{
Anjali Bansal Gupta and Ramasubbu Sankararamakrishnan*
}

Address: Department of Biological Sciences and Bioengineering, Indian Institute of Technology Kanpur, Kanpur 208016, India

Email: Anjali Bansal Gupta - anjalib@iitk.ac.in; Ramasubbu Sankararamakrishnan* - rsankar@iitk.ac.in

* Corresponding author

Published: 20 November 2009

BMC Plant Biology 2009, 9:134 doi:10.1 186/147/-2229-9-134
Received: 3 September 2008

Accepted: 20 November 2009

This article is available from: http://www.biomedcentral.com//47/-2229/9//34

(c) 2009 Gupta and Sankararamakrishnan; licensee BioMed Central Ltd.

This is an Open Access article distributed under the terms of the Creative Commons Attribution License (http://creativecommons.org/licenses/by/2.0), which permits unrestricted use, distribution, and reproduction in any medium, provided the original work is properly cited.

\begin{abstract}
Background: Members of major intrinsic proteins (MIPs) include water-conducting aquaporins and glycerol-transporting aquaglyceroporins. MIPs play important role in plant-water relations. The model plants Arabidopsis thaliana, rice and maize contain more than 30 MIPs and based on phylogenetic analysis they can be divided into at least four subfamilies. Populus trichocarpa is a model tree species and provides an opportunity to investigate several tree-specific traits. In this study, we have investigated Populus MIPs (PtMIPs) and compared them with their counterparts in Arabidopsis, rice and maize.
\end{abstract}

Results: Fifty five full-length MIPs have been identified in Populus genome. Phylogenetic analysis reveals that Populus has a fifth uncharacterized subfamily (XIPs). Three-dimensional models of all 55 PtMIPs were constructed using homology modeling technique. Aromatic/arginine (ar/R) selectivity filters, characteristics of loops responsible for solute selectivity (loop C) and gating (loop D) and group conservation of small and weakly polar interfacial residues have been analyzed. Majority of the non-XIP PtMIPs are similar to those in Arabidopsis, rice and maize. Additional XIPs were identified from database search and 35 XIP sequences from dicots, fungi, moss and protozoa were analyzed. Ar/R selectivity filters of dicots XIPs are more hydrophobic compared to fungi and moss XIPs and hence they are likely to transport hydrophobic solutes. Loop $C$ is longer in one of the subgroups of dicot XIPs and most probably has a significant role in solute selectivity. Loop D in dicot XIPs has higher number of basic residues. Intron loss is observed on two occasions: once between two subfamilies of eudicots and monocot and in the second instance, when dicot and moss XIPs diverged from fungi. Expression analysis of Populus MIPs indicates that Populus XIPs don't show any tissue-specific transcript abundance.

Conclusion: Due to whole genome duplication, Populus has the largest number of MIPs identified in any single species. Non-XIP MIPs are similar in all four plant species considered in this study. Small and weakly polar residues at the helix-helix interface are group conserved presumably to maintain the hourglass fold of MIP channels. Substitutions in ar/R selectivity filter, insertion/deletion in loop $C$, increasing basic nature of loop $D$ and loss of introns are some of the events occurred during the evolution of dicot XIPs. 


\section{Background}

Water transport in different parts of a plant is significantly contributed by the integral membrane channel protein, aquaporin, which is a member of the Major Intrinsic Protein (MIP) superfamily [1]. In addition to their role in plant soil-water relations [2,3], members of this family are also implicated in plant reproduction [4,5], cell elongation [6], plant cell osmoregulation [7] and seed germination [8]. Aquaporins also influence leaf physiology and leaf movements $[9,10]$, drought resistance [11], salt tolerance $[12,13]$ and fruit ripening [14] in plants. MIP family consists of both aquaporins [15] and aquaglyceroporins $[16,17]$. A large number of MIP genes have been identified in plants and they seem to be diverse. Arabidopsis [18], maize [19] and rice [20,21] each have more than $30 \mathrm{MIP}$ genes. Phylogenetic analysis reveals that the MIP genes can be largely divided into at least four different subfamilies and they have been classified as plasma membrane intrinsic proteins (PIPs), tonoplast intrinsic proteins (TIPs), nodulin-26 intrinsic proteins (NIPs) and small basic intrinsic proteins (SIPs) $[18,19,21,22]$. Three additional subfamilies have been recently reported. In the nonvascular moss Physcomitrella patens which is a primitive land plant, a novel plant MIP (GIP) homologous to bacterial glycerol channels found in gram-positive bacteria has been identified [23]. Two other subfamilies found recently in the same species are hybrid intrinsic proteins (HIPs) and unrecognized X intrinsic proteins (XIPs) [24]. Substrate specificity, expression and localization of many members of PIPs, TIPs and NIPs have been investigated. Plant MIPs localize in plasma membranes (PIPs and some NIPs) [25-27], tonoplast (TIPs) [28], endoplasmic reticulum (SIPs) [29] and other subcellular compartments [30]. In addition to water and glycerol [31-33], PIPs, TIPs and NIPs facilitate the transport of other unconventional neutral solutes and gases [34]. This includes urea [35-37], lactic acid [38] and metalloids like boron [27,39], silicon [26], arsenic and antimony [40,41]. Carbon dioxide [42], hydrogen peroxide [43] and NH3 [44,45] are among the other molecules that are transported by plant MIPs. The transport activity of these MIP genes is regulated by many factors including cotranslational and post-translational modifications [46-48], gating [49] or subcellular trafficking $[50,51]$. Members of XIPs and HIPs are the least characterized and they need further investigation regarding solute transport, expression and other properties.

Three-dimensional structures of proteins belonging to MIP family have been determined from several organisms [52-58] including a plant aquaporin SoPIP2;1 from spinach [49]. All MIP structures exhibit a conserved hourglass fold with $\alpha$-helical bundle comprising six transmembrane (TM) helices (H1 to H6) and two half-helices. The halfhelices forming the seventh TM helix are from loops B and $\mathrm{E}$ (LB and LE) that also possess the signature sequence
Asn-Pro-Ala (NPA). These conserved motifs from the two half-helices meet approximately at the center of the membrane giving rise to one of the two pore constrictions. The second constriction, also known as aromatic/arginine (ar/ R) selectivity filter, is formed by four residues towards the extracellular side approximately $8 \AA$ from the NPA region. The four residues in this selectivity filter are contributed by transmembrane helices H2, H5 and the loop LE. Molecular mechanism of water and glycerol transport, exclusion of charged groups and specificity of solute transport have been investigated by computational [59-63] and experimental studies [64,65]. Recently, homology modeling was carried out on Arabidopsis, rice and maize MIPs [21,66] and the structures were classified based on the residues in the ar/ $\mathrm{R}$ selectivity filter. The diversity of pore configurations indicated that the plant MIPs could transport much more diverse solutes than their counterparts in mammals.

The genome sequence of the model tree plant Populus trichocarpa (Black cottonwood) has been recently determined [67]. Phylogenetically, Populus is more closely related to Arabidopsis than the model cereal plant rice. Populus is a eudicot and both Populus and Arabidopsis are clustered in angiosperm Euroside I clade [68]. The availability of genomes of Arabidopsis, Populus and rice will facilitate the study of comparative biology of all the three species. As a second eudicot genome sequence with its modest genome size, Populus trichocarpa offers unique opportunity to study some aspects that cannot be studied in other model annual plants [68]. Examples include wood development, seasonality, flowering and natural variation [69]. Apart from its genomic sequence, other Populus genomic resources such as Populus EST sequences, full-length cDNA sequences and DNA microarrays also offer tools to study Populus biology [70-72]. Populus is also a good model system in which long distance transport of water and nutrients can be investigated. However, there are only few studies on poplar aquaporins and their role in long distance transport of water and other nutrients. Seven aquaporins have been investigated in mycorrhized poplar plants and it has been shown that there is a strong increase in the capacity of water transport in plasma membrane of root cells [73]. Analysis of EST sequences from the root of hybrid cottonwood described the expression levels of Populus PIP and TIP members during different stages of adventitious root development [74]. A recent study by Danielson and Johanson [24] identified a group of aquaporins from Populus belonging to the unrecognized XIP category. As in Arabidopsis and rice, the availability of Populus genome sequence gives an opportunity to identify and characterize the whole repertoire of MIPs in this species. In this paper, we have carried out genome-wide analysis of Populus MIPs from its genomic sequence and characterized them. We have identified 55 full-length MIP 
genes in Populus and this is the largest number of MIP genes identified in any single species to date. We have compared several features of Populus MIPs with their counterparts in Arabidopsis, rice and maize. The unique features identified in Populus MIPs are discussed in this paper.

\section{Results \\ MIP genes in Populus genome}

The whole genome shotgun (WGS) sequence of Populus trichocarpa [67] available at NCBI [75] was searched using TBLASTN [76] for genes coding for MIPs. The initial query sequence from rice OsPIP2; 1 resulted in identification of 41 Populus MIPs (PtMIPs). Five other query sequences representing PIP, TIP, NIP, SIP and XIP family members from the initial search results yielded additional MIP proteins. A list of more than 50 full-length MIP proteins from Populus WGS contigs was obtained (Table 1) after discarding those sequences with missing transmembrane regions or interrupted by a stop codon in the middle of the sequence as predicted by the program GeneMark $[77,78]$. The Populus genome paper [67] has reported 67 genes belonging to major intrinsic protein family (Table S12 in the reference Tuskan et al. [67]), although the details are not mentioned. The Joint Genome Institute (JGI) has listed 63 aquaporin genes (KOG ID: 0223) belonging to Populus trichocarpa. We have carefully compared the MIP proteins from our TBLASTN search result with those 63 from JGI and found that there are 50 sequences common between both of them. We find that 9 of the 63 MIP proteins from JGI have to be discarded for various reasons (Additional file 1: Table S1). Four JGI sequences were not found in our search. One sequence from our search (NCBI accession no. AARH01008299) is not present in the JGI list. Thus, we have finally obtained 55 full-length MIP protein sequences from Populus trichocarpa which is the largest set of MIP sequences from any single species identified so far and they are listed in Table 1 . The available data shows that forty four Populus MIP genes are nearly uniformly spread over 13 of the 19 haploid chromosomes. Nine out of 13 chromosomes have at least 3 MIPs each with the highest number of eight MIPs observed in chromosome IX (Table 1). The remaining genes are located on a scaffold not yet assigned to a chromosome.

\section{Comparison of Populus MIPs with MIPs of Arabidopsis, rice and maize}

PtMIPs were compared individually with MIPs from Arabidopsis (AtMIPs), rice (OsMIPs) and maize (ZmMIPs). Then all MIPs from the four plant species were compared together. Multiple sequence alignments of full length proteins using the program T-COFFEE [79] were generated on different sets of MIP sequences, namely (i) PtMIPs, (ii) PtMIPs and AtMIPs, (iii) PtMIPs and OsMIPs, (iv) PtMIPs and ZmMIPs and (v) PtMIPs, AtMIPs, OsMIPs and
ZmMIPs. The trees created using these alignments by neighbor-joining (NJ) method shows that PtMIPs can be classified into five subfamilies. PIPs, TIPs, NIPs and SIPs from Populus clustered with the respective subfamilies from Arabidopsis, rice and maize (Figure 1, Additional files 2 to 4 ). The fifth subfamily belongs to the uncharacterized XIP family and is not observed in the other three plant species. Sequences belonging to neither HIP nor GIP family $[23,24]$ are found in all the four plant species. When MIPs from all four plant species were considered together, the corresponding non-XIP subfamily members clustered together and XIPs observed only in Populus clustered separately (Additional file 4). The results of NJ method were found to be very similar to those by heuristic distance, parsimony and maximum likelihood methods with the clustering more or less maintained in all three methods (data not shown). Among the 55 PtMIPs, there are 15 PIPs, 17 TIPs, 11 NIPs, 6SIPs and 6XIPs. Both PIPs (15 PtPIPs vs 13 in other plants) and NIPs (11 PtNIPs vs. 9 to 13 in Arabidopsis and rice) are similar in number found in other plants. The expression of most of the PtPIP and PtTIP sequences are supported by the Populus EST sequences (Table 1). The increase in the number of PtMIPs can be attributed to the increase in the number of PtTIPs and PtSIPs and also the presence of a new XIP subfamily with 6 members. The other three plants have 10 to 11 TIPs and 2 to 3 SIPs. The additional 15 PtMIPs belonging to TIP, SIP and XIP subfamilies explain the largest number of MIPs observed in Populus.

Each subfamily was further subdivided into groups according to their clustering in the phylogenetic tree and their similarity with the known MIPs from other plants. As in other plants, Populus PIPs and TIPs have two (PtPIP1 and PtPIP2) and five (PtTIP1 to PtTIP5) subgroups respectively. However, maximum number of seven subgroups is observed for Arabidopsis NIPs while Populus, maize and rice NIPs have only three to four subgroups. Two PtNIP members (PtNIP3;1 and PtNIP3;2) have substitutions in both NPA motifs. Although, two subgroups are observed for PtSIP subfamily similar to other plants under study, the number of SIP proteins found in Populus is the maximum observed so far. The Ala residue in the first NPA motif in four out of 6 PtSIPs is substituted by Thr or Leu. The uncharacterized XIP family found only in Populus among the four species has two subgroups PtXIP1 and PtXIP2. While sequences from other subfamilies have been analyzed and studied experimentally, little is known about the XIP family members. We have identified additional members of XIP family and further sequence analysis and homology modeling helped us to characterize this subfamily further and the details are explained below. 
Table I: MIP genes in Populus genome identified from the NCBI whole genome shotgun contigs.

\begin{tabular}{|c|c|c|c|c|c|c|}
\hline Name & $\begin{array}{l}\text { NCBI WGS contig } \\
\text { accession number }\end{array}$ & $\begin{array}{l}\text { JGI accession } \\
\text { number }\end{array}$ & EST accession & NPA motif (LB) ${ }^{a}$ & NPA motif (LE) $)^{a}$ & $\underset{\text { tione }}{\text { Chromosome loca- }}$ \\
\hline PtPIPI;I & AARHOI004386 & 724520 & DT472648 & - & - & $x$ \\
\hline PtPIPI;2 & AARHOI00354I & 656216 & BU868I42 & - & - & VIII \\
\hline PtPIPI;3 & AARHOI00I832 & 711735 & DT474III & - & - & III \\
\hline PtPIPI;4 & AARHOI003029 & 831918 & & - & - & VI \\
\hline PtPIPI;5 & AARHOI006875 & 835561 & & - & - & $\mathrm{XVI}$ \\
\hline PtPIP2;I & AARHOI003794 & 821084 & DT478685 & - & - & IX \\
\hline PtPIP2;2 & AARHOI00254I & 648808 & DT4735I3 & - & - & IV \\
\hline PtPIP2;3 & AARHOI0044I2 & 567607 & DT474367 & - & - & $x$ \\
\hline PtPIP2;4 & AARHOI003539 & 563742 & DT472264 & - & - & VIII \\
\hline PtPIP2;5 & AARHOIO08299 & 826419 & BU868919 & - & - & $*$ \\
\hline PtPIP2;6b & AARHOI008299 & N/A & CV227359 & - & - & $*$ \\
\hline PtPIP2;7 & AARHOI00675 I & 735495 & DT4988I5 & - & - & $\mathrm{XVI}$ \\
\hline PtPIP2;8 & AARHOI0039/2 & 821627 & & - & - & IX \\
\hline PtPIP2;9c & & 836572 & & - & - & $*$ \\
\hline PtPIP2; 10 & AARHOI008738 & 796664 & & - & - & $*$ \\
\hline PtTIPI;I & AARHOI000429 & 549212 & & - & - & 1 \\
\hline PtTIPI;2 & AARHOI003864 & 833283 & DT499779 & - & - & IX \\
\hline PtTIPI;3 & AARHOI004405 & 822504 & DT476627 & - & - & $x$ \\
\hline PtTIPI;4 & AARHOI 003540 & 656044 & BU875073 & - & - & VIII \\
\hline PtTIPI;5 & AARHOI006799 & 667870 & DT497619 & - & - & $\mathrm{XVI}$ \\
\hline PtTIPI;6 & AARHOI008323 & 589502 & DT497300 & - & - & $*$ \\
\hline PtTIPI;7 & AARHOI003929 & 558321 & & - & - & IX \\
\hline PtTIPI;8 & AARHOIOI0693 & 828458 & & - & - & $*$ \\
\hline PtTIP2;I & AARHOI000349 & 548890 & DT496472 & - & - & 1 \\
\hline PtTIP2;2 & AARHOI00I73I & 645978 & DT488368 & - & - & III \\
\hline PtTIP2;3 & AARHOIO0I777 & 817166 & & - & - & III \\
\hline PtTIP2;4 & AARHOI008349 & 676397 & & - & - & $*$ \\
\hline PtTIP3;I & AARHOIOI070I & 584517 & & - & - & $*$ \\
\hline
\end{tabular}


Table I: MIP genes in Populus genome identified from the NCBI whole genome shotgun contigs. (Continued)

\begin{tabular}{|c|c|c|c|c|c|c|}
\hline PtTIP3;2 & AARHOIOII328 & 811826 & & - & - & $*$ \\
\hline PtTIP4;I & AARHOI003I45 & 561759 & CV233830 & - & - & $\mathrm{VI}$ \\
\hline PtTIP5; Id & AARHOI00I826 & 414059 & & - & - & III \\
\hline PtTIP5;2d & AARHOIO08384 & 423803 & & - & - & $*$ \\
\hline PtNIPI; Id & AARHOI002I08 & 197507 & & - & - & IV \\
\hline PtNIPI; $2^{d}$ & AARHOI004854 & 235172 & & - & - & $X I$ \\
\hline PtNIPI;3 & AARHOI004327 & 566501 & & - & - & $x$ \\
\hline PtNIPI;4c & & 756079 & & - & - & II \\
\hline $\mathrm{PtNIPI} ; 5^{\mathrm{c}}$ & & 754717 & & - & - & II \\
\hline PtNIP2; I & AARHOI007I72 & 577637 & & - & - & XVII \\
\hline PtNIP3; I & AARHOI00I86I & 757987 & & NPS & NPV & III \\
\hline PtNIP3;2 & AARHOIO0OIIO & $797 \mid 36$ & & NPS & NPV & 1 \\
\hline PtNIP3;3 & AARHOI000968 & 708017 & DT488082 & - & - & 1 \\
\hline PtNIP3;4 & AARHOI005007 & 823094 & & - & - & $X I$ \\
\hline PtNIP3;5 & AARHOI00369I & 803915 & & - & - & VIII \\
\hline PtSIPI;I & AARHOIO05657 & 729942 & & NPT & - & XIII \\
\hline PtSIPI;2 & AARHOI007966 & 665418 & DT487219 & NPT & - & XIX \\
\hline PtSIPI;3 & AARHOIOOI 280 & 755885 & & - & - & II \\
\hline PtSIPI;4c & & 572968 & & - & - & XIX \\
\hline PtSIP2;I & AARHOI00662I & 734665 & DN491635 & NPL & - & $X V I$ \\
\hline PtSIP2;2 & AARHOIO02935 & 652505 & & NPL & - & $\mathrm{VI}$ \\
\hline PtXIPI;I & AARHOIO22440 & 829126 & & NPI & - & $*$ \\
\hline PtXIPI;2 & AARHOIO03797 & 557139 & & NPI & - & IX \\
\hline $\mathrm{PtXIPI;3}$ & AARHOIO02537 & 759781 & & NPI & - & IV \\
\hline PtXIPI;4 & AARHOIO03797 & 767334 & & NPL & - & IX \\
\hline PtXIPI;5 & AARHOIO03797 & 821124 & & - & - & IX \\
\hline PtXIP2;I & AARHOI003797 & 557138 & & SPV & - & IX \\
\hline
\end{tabular}

aDeviation of NPA signature motif in loops B and E is reported. '-' indicates that NPA motif is conserved.

bThis gene is not listed in JGI. The coding regions in the NCBI WGS contig are 173376-173676, I738II-174106, 174224-174364, 174465-174584.

cFour PtMIP genes are not found in our TBLASTN search of NCBI database. Their JGI accession codes are given.

dThe sequences for these genes predicted by GeneMark are longer than those found in JGI. GeneMark-predicted sequences have extensions in N-

or C-terminus. In this study, we have used GeneMark predicted sequences due to their similarity with Arabidopsis sequences

eThe symbol ' $*$ ' indicates that these genes are located on a scaffold not yet assigned to a chromosome. 


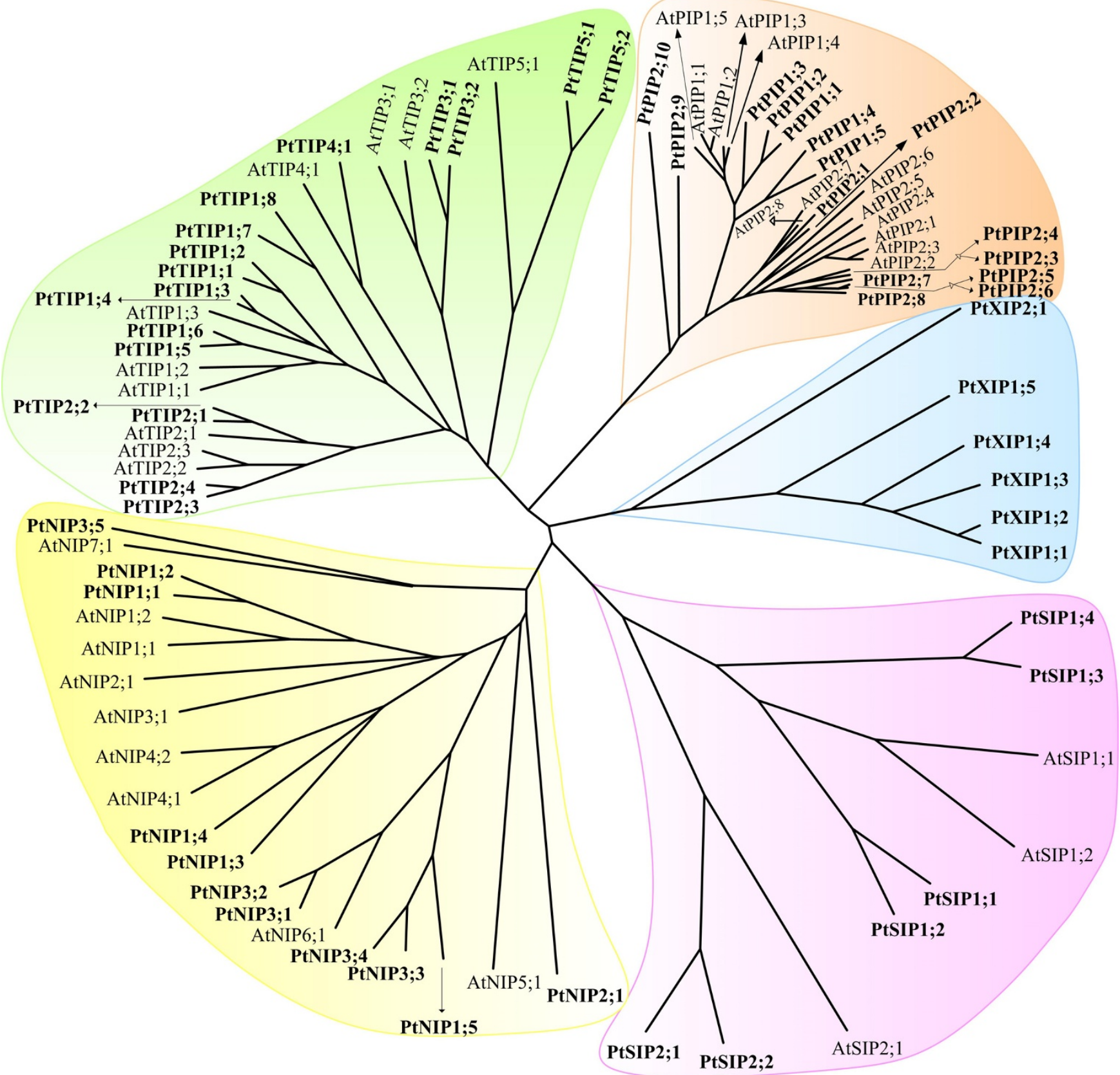

Figure I

Evolutionary relationship of Populus MIPs. Phylogenetic analysis of all Populus MIPs is shown along with MIPs from Arabidopsis. Neighbor-Joining (NJ) method was used to create this unrooted tree. NJ method used the multiple sequence alignment generated by T-COFFEE to generate the tree. Populus MIP subfamilies PtPIPs, PtTIPs, PtNIPs and PtSIPs clustered with the corresponding Arabidopsis MIP subfamilies. XIPs observed only in Populus clearly form a separate group. Each MIP subfamily is shown with a specific background color to distinguish them from others. A similar result is obtained when the same analysis was carried out with rice and maize MIPs (Additional files 2 to 4). 


\section{XIP subfamily members in other species}

Danielson and Johanson [24] have reported 19 XIP members that included 5 Populus XIPs. Among the XIPs, 10 were from dicot plants other than Populus, three were from moss and one was from a protozoa. No XIP homolog was found in monocots. We examined all these sequences and found that the sequence from Nicotiana benthamina (GenBank ID: CK295158) lacks the first transmembrane segment. Similarly, one of the EST sequences for Liriodendron tulipifera (GenBank ID: DT60037) is lacking NCBI record. Hence, these two sequences were discarded for further analysis of XIP sequences. In addition to the 6 Populus XIPs identified in the present study ( 5 of them have been reported by Danielson and Johanson [24] also), we have considered the 12 additional XIP sequences from plants, moss and the protozoa reported earlier [24].

In order to identify additional XIP members, we used each of the six PtXIP sequence as a query and searched the plant EST databases using TBLASTN [80]. We have identified an additional 8 XIP sequences from dicot plants (Table 2). We also carried out TBLASTN searches on various completed and partial genome sequences of different organism groups available in NCBI. To our surprise, many hits were obtained from organisms that are classified as fungi with e-values ranging from $4.0 \mathrm{E}-17$ to $1.0 \mathrm{E}-04$. The program GeneMark $[77,78]$ was used to identify the coding regions and we found 9 full-length (Table 3 ) and 5 partial fungi MIP sequences based on GeneMark predictions. Partial fungi sequences were not considered for further analysis (Additional file 1: Table S2). Thus our search of plant EST database and fungi genomic sequences yielded another additional 17 XIP sequences. Taken together, we have considered 6 Populus XIPs, 16 XIPs from other dicot plants, 9 fungi XIPs, 3 moss XIPs and 1 protozoan XIP (Total 35 XIPs) for further analysis.

We have carried out phylogenetic analysis of all PtMIPs along with all XIPs. The XIPs from fungi, other dicot plants and moss clustered together with PtXIPs and are grouped separately from other Populus subfamilies namely PtPIPs, PtTIPs, PtNIPs and PtSIPs (Additional file 5). When only XIP members are considered, the fungi and moss XIPs form two independent clusters separate from the dicot XIPs (Figure 2). All the dicot XIPs fall into one of the two subgroups, XIP1 or XIP2. The lone XIP from protozoa does not fall into any of the four groups. Analysis of pairwise sequence alignments indicates that the XIP sequences within the subgroup are highly similar. The average sequence identities between pairs of sequences within XIP1 and XIP2 groups are $71 \%$ and $~ 70 \%$ respectively (Table 4). However, the sequence variation between the two XIP groups is significant and the average sequence identity falls to $\sim 40 \%$ when sequences are compared across the two groups. XIPs of dicot plants have diverged from those sequences from fungi and moss. The range of average sequence identities between plant XIPs and fungi/ moss XIPs varies from $\sim 27 \%$ to $34 \%$. Among the fungi XIPs, some pairs of sequences are very similar. For example, XIP sequences from Fusarium oxysporum and Gibberella moniliformis have $~ 94 \%$ sequence identity. When all 36 possible fungi XIP pairs from 9 sequences are considered, the average pairwise sequence identity is only $\sim 47 \%$. However, there are four pairs within fungi XIPs whose sequence identity exceeds $70 \%$. When PtXIPs are compared with other MIP subfamilies in Populus, namely PtPIPs, PtNIPs, PtTIPs and PtSIPs, the average pairwise sequence identities vary from 25 to $32 \%$. This indicates

Table 2: Additional dicot XIPs identified from TBLASTN search of plant EST databases.

\begin{tabular}{lllll}
\hline XIP Name & Species name & EST accession number & NPA motif (LB) & NAP motif (LE) $^{\mathbf{b}}$ \\
\hline LsXIPI;I & Lactuca sericola & DWI09344 & - & - \\
\hline CcXIPI;2 & Citrus climentina & DY262747 & NPL & - \\
\hline CcXIPI;3 & Citrus climentina & DY263320 & NPL & - \\
\hline CcXIPI;4 & Citrus climentina & DY256802 & NPL & - \\
\hline CsXIPI;1 & Citrus sinensis & CX049225 & NPL & - \\
\hline CsXIPI;2 & Citrus sinensis & CX049224 & NPL & - \\
\hline RcXIP2;2 & Ricinus communis & EG664940 & SPT & -
\end{tabular}

aCcXIPI;I and RcXIP2;I are the XIPs from the respective species reported in Danielson and Johanson [24]. See also Figure 2.

bDeviation of NPA signature motif in loops B and E is reported. '-' indicates that NPA motif is conserved. 
Table 3: XIPs identified from the genomic sequences of fungi

\begin{tabular}{|c|c|c|c|c|}
\hline Name $^{a}$ & Species name & $\begin{array}{l}\text { NCBI accession number } \\
\text { [Coding regions] }\end{array}$ & NPA motif (LB) ${ }^{b}$ & NPA motif (LE) ${ }^{b}$ \\
\hline F-PmXIP & Penicillium marneffei & $\begin{array}{l}\text { ABAR0I000036 } \\
{[47839-48091,48166-48854]}\end{array}$ & NPT & - \\
\hline F-TsXIP & Talaromyces stipitatus & $\begin{array}{l}\text { ABASOI0000I3 } \\
{[I 78746-I 78668, I 78588-I 78022, I 77946-177870,} \\
I 77773-177696]\end{array}$ & NPT & - \\
\hline F-TvXIPI & Trichoderma virens & $\begin{array}{l}\text { ABDF0I000006 } \\
{[3 / 7889-3 / 81 / 9,3 / 82 / 5-3 / 8437,3 / 8628-3 / 87 / 5} \\
3 / 8809-3 / 9092,3 / 9203-3 / 9360]\end{array}$ & NPM & NPS \\
\hline F-TvXIP2 & Trichoderma virens & $\begin{array}{l}\text { ABDF0I0002I5 } \\
{[85889-86 / 54,863 / 3-86842,86893-87062]}\end{array}$ & - & - \\
\hline F-FoXIP & Fusarium oxysporum f. sp. Lycopersici & $\begin{array}{l}\text { AAXHOI0007I6 } \\
{[22960-22681,22584-21929]}\end{array}$ & NPL & - \\
\hline F-GmXIP & Gibberella moniliformis & $\begin{array}{l}\text { AAIM02000I33 } \\
{[4780 I-47522,47424-46769]}\end{array}$ & NPL & - \\
\hline F-TaXIP & Trichoderma atroviride & $\begin{array}{l}\text { ABDG0I000060 } \\
{[27703-27452,27334-27 / \mid 2,2696 /-26874,268 / 5-} \\
26532,26467-26289]\end{array}$ & NPT & - \\
\hline F-TrXIP & Trichoderma reesei & $\begin{array}{l}\text { AAILOI000I83 } \\
{[17864-18130,18289-18511,18709-18796,18890-} \\
19173,19303-19460]\end{array}$ & NPM & NPS \\
\hline F-AtXIP & Aspergillus terreus & $\begin{array}{l}\text { AAJNOI } 000055 \\
{[2572 I-25970,26017-26595,26663-2675 I]}\end{array}$ & SPT & - \\
\hline
\end{tabular}

aThe names are prefixed with "F-" to distinguish fungi XIPs from other XIPs.

bDeviation of NPA signature motif in loops B and E is reported. '-' indicates that NPA motif is conserved.

that PtXIPs have diverged significantly from other subfamilies. Notably, substitutions are observed in the conserved NPA motif in loop B in almost all XIPs. However, the recent crystal structure of an MIP homolog from Plasmodium falciparum [58] in which both the NPA motifs are substituted, indicates that the mutations in the conserved in NPA motif are compensated by covariant mutations throughout the protein.

Comparison of ar/R selectivity filters in PtMIPs and XIPs Knowledge of three-dimensional structure helps to understand the mechanism of a protein's function at molecular

Table 4: Average pairwise sequence identities (in percentage) of XIP subfamilies from different organism groups

\begin{tabular}{|c|c|c|c|c|c|}
\hline & $\begin{array}{l}\text { Dicot XIP Is } \\
(18)^{\mathrm{a}}\end{array}$ & $\begin{array}{l}\text { Dicot XIP2s } \\
(4)^{\mathrm{a}}\end{array}$ & $\begin{array}{l}\text { Moss XIPs } \\
(3)^{\mathrm{a}}\end{array}$ & $\begin{array}{l}\text { Fungi XIPs } \\
(9)^{\mathrm{a}}\end{array}$ & $\begin{array}{l}\text { Protozoa } \\
\text { (I) }\end{array}$ \\
\hline Dicot XIPIs & 70.6 & & & & \\
\hline Dicot XIP2s & 39.9 & 69.5 & & & \\
\hline mossXIPs & 34.3 & 32.0 & 33.9 & & \\
\hline Fungi XIPs & 32.0 & 27.1 & 30.1 & 47.2 & \\
\hline Protozoa & 32.3 & 29.1 & 27.8 & 27.8 & \\
\hline
\end{tabular}

aThe number of sequences under each group is given in brackets. 


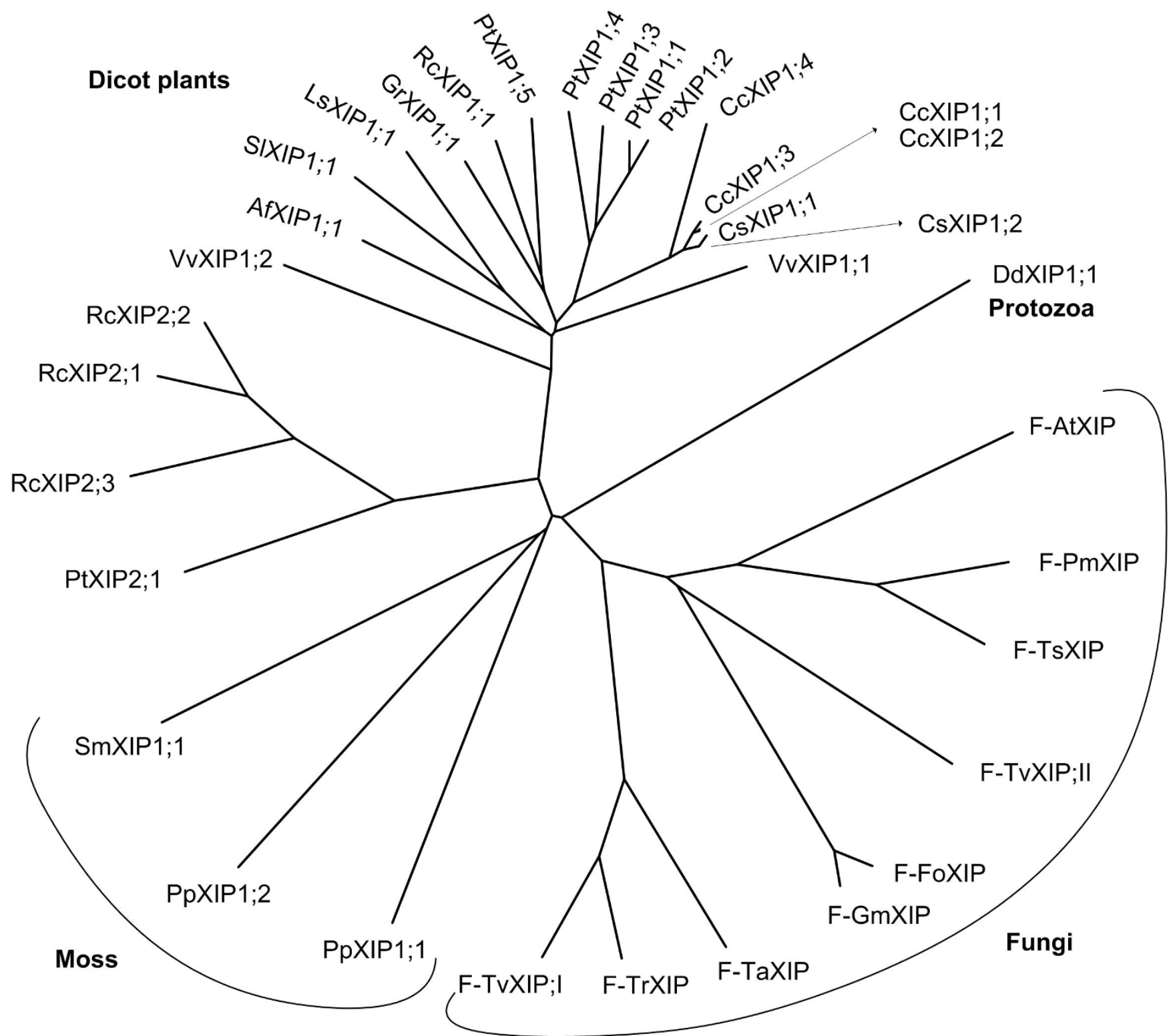

0.1

Figure 2 (see legend on next page) 
Figure 2 (see previous page)

Phylogenetic analysis of XIPs. All 35 XIPs from dicot plants, fungi, moss and protozoa have been used to construct the phylogenetic tree using NJ method. Multiple sequence alignment for creating the phylogenetic tree was generated by T-COFFEE. XIPs from dicot plants, fungi and moss cluster separately. All dicot XIPs cluster into two subgroups XIPI and XIP2. All fungi XIPs and some dicot XIPs (Table 2) have been identified in this study. Other dicot XIPs, moss XIPs and the lone XIP from protozoa were identified by Danielson and Johanson. The names of Populus XIPs, dicot XIPs identified in this study and fungi XIPs are given in Tables I, 2 and 3 respectively. The names of other dicot and the protozoan XIPs are given as follows with their IDs (EST/RefSeq/whole genome shotgun sequence) in brackets: SIXIPI;I -- Solanum lycopersicum (BT0I4I97), CcXIPI; I -Citrus clementina (DY275505), GrXIPI;I -- Gossypium raimondi (CO092422), RcXIPI;I -- Ricinus communis (EG656577), RcXIP2; I -- Ricinus communis (EG666650), AfXIPI;I -- Aquilegia formosa $\times$ Aquilegia pubescens (DR936893 and DT742029), DdXIPI;I -- Dictyostelium discoideum (XM_639I70) and VvXIPI;I and VvXIPI;2 -- Vitis vinifera (AM455454).

level. To date, the structure of only one plant MIP protein (SoPIP2;1) has been determined experimentally at atomic level [49]. Homology modeling technique has been used to build three-dimensional models of plant MIPs and it helped to identify different structural subclasses based on the residues in the ar/R selectivity filter $[21,66]$. Such an approach also helped to identify the group conservation of small/weakly polar residues at the helix-helix interface. We have modeled all the PtMIP proteins and the additional XIPs found in other dicot plants, fungi, moss and protozoa. We have analyzed the ar/R selectivity filters of all PtMIPs with a specific focus to XIP proteins. The nonXIP proteins from Populus have been compared with those from XIPs. Our structure-based sequence alignments of PtMIPs and XIPs help us to identify features in XIP proteins that distinguish them from MIPs from other subfamily.

Analysis of ar/R selectivity filters in PtPIPs, PtTIPs, PtNIPs and PtSIPs indicate that residues forming the selectivity filter region are very similar to their counterparts in other three plants compared in this study. Only three out of 49 non-XIPs show some distinct features in this region (Table $5)$. With the lone exception of PtPIP2;10, all PIPs from Arabidopsis, rice and maize and 14 out of 15 PtPIPs have Phe from helix H2, His from helix H5, Thr and Arg from loop E (LE1 and LE2 positions) forming the ar/R selectivity region. PtPIP2;10 has Asn in the place of Phe in $\mathrm{H} 2$ position making the pore constriction more hydrophilic (Figure 3A). Among the PtNIPs, PtNIP1;5 is somewhat similar to the other members of PtNIP1 subgroup. However, it has two small residues in positions H5 and LE1 making the size of the constriction at this point relatively larger. Similarly, PtSIP1; 1 has a unique substitution in the $\mathrm{ar} / \mathrm{R}$ tetrad in which the conserved Arg in loop $\mathrm{E}$ is replaced by bulky hydrophobic Phe. With the other three positions occupied by hydrophobic residues (Ile in $\mathrm{H} 2$, Val in H5 and Pro in LE1), this could be one of the most hydrophobic pore constriction in the MIP members (Figure $3 \mathrm{~B}$ ). Overall our results suggest that majority of PtMIPs that are not XIPs have ar/R signatures similar to the ones present in Arabidopsis, rice and maize. This might be an indication that these MIPs from Populus facilitate the transport of same or similar solute molecules that are transported in other plants. In other words, only a couple of PtMIPs belonging to the four well known subfamilies could be involved in the transport of novel solute molecules that may be considered unique to Populus.

Analysis of three-dimensional models of PtXIPs and other XIPs indicate that the features observed in ar/R selectivity filters are distinct in some XIPs. Among all the XIPs, dicot plant XIPs differ from those XIPs from moss and fungi. XIPs from dicots can be divided into four structural subclasses based on ar/R signatures (Table 6). In the first group, thirteen XIP sequences have Val/Ile (H2), Thr (H5), Ala (LE1) and Arg (LE2) as ar/R signature. This is

Table 5: Ar/R selectivity filters of Populus PIP, TIP, NIP and SIP members

\begin{tabular}{|c|c|c|c|c|}
\hline MIP Members ${ }^{a}$ & H2 & H5 & LEI & LE2 \\
\hline \multicolumn{5}{|l|}{ PIP family } \\
\hline $\begin{array}{l}\text { PtPIPI;I to PtPIPI;5 } \\
\text { PtPIP2;I to PtPIP2;9 }\end{array}$ & $\mathrm{F}$ & $\mathrm{H}$ & $\mathrm{T}$ & $\mathrm{R}$ \\
\hline PtPIP2; 10 & $N$ & $H$ & $T$ & $R$ \\
\hline \multicolumn{5}{|l|}{ TIP family } \\
\hline PtTIPI;I to PtTIPI;8 & $\mathrm{H}$ & 1 & $A$ & V \\
\hline PtTIP2; I to PtTIP2;4 & $\mathrm{H}$ & 1 & G & $\mathrm{R}$ \\
\hline PtTIP3;I, PtTIP3;2, PtTIP4;। & $\mathrm{H}$ & I & $A$ & $\mathrm{R}$ \\
\hline PtTIP5;I, PtTIP5;2 & $\mathrm{N}$ & $\mathrm{V}$ & G & C \\
\hline \multicolumn{5}{|l|}{ NIP family } \\
\hline PtNIPI;I to PtNIPI;4 & W & V & A & $\mathrm{R}$ \\
\hline PtNIPI;5 & W & $A$ & $A$ & $R$ \\
\hline PtNIP2; I & G & $\mathrm{S}$ & G & $\mathrm{R}$ \\
\hline PtNIP3; I to PtNIP3;5 & S/T/A & $\mathrm{I} / \mathrm{V}$ & $\mathrm{G} / \mathrm{A}$ & $\mathrm{R}$ \\
\hline \multicolumn{5}{|l|}{ SIP family } \\
\hline$P t S I P I ; I$ & 1 & $V$ & $P$ & $F$ \\
\hline PtSIPI;2, PtSIPI;3, PtSIPI;4 & V/A & V & $P$ & $\mathrm{~N}$ \\
\hline PtSIP2; I, PtSIP2;2 & $\mathrm{T} / \mathrm{S}$ & $\mathrm{H}$ & G & $S$ \\
\hline
\end{tabular}

aPtMIP members and the corresponding selectivity filter residues are shown in italics if the ar/R selectivity filter is not found in Arabidopsis, rice or maize. 

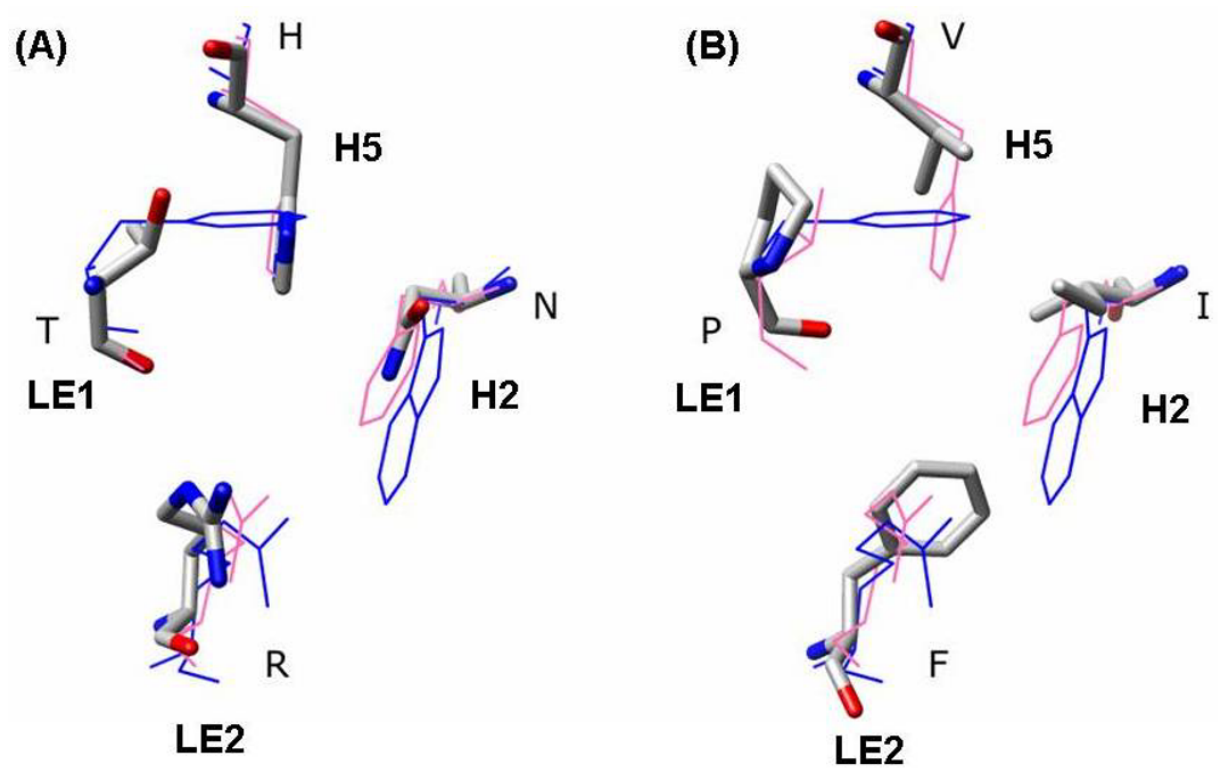

\section{Figure 3}

Ar/R selectivity filters of PtPIP2; 10 and PtSIP I; I. Ar/R selectivity filters of non-XIP Populus MIPs that are not found in their counterparts from Arabidopsis, rice and maize. Transmembrane regions of the MIP models from Populus were superposed on the experimentally determined structures of glycerol transporter GlpF (PDB ID: IFX8) and the water-transporting spinach aquaporin SoPIP2; I (PDB ID: IZ98). Populus MIP residues are shown in stick representation with nitrogen and oxygen atoms in blue and red colors respectively. PtMIP residues are displayed in one letter code and their corresponding positions in the selectivity filter are indicated. For comparison purpose, the ar/R filters of GlpF and SoPIP2;I are also shown in blue and pink respectively. (A) Out of 54 PIPs from four plants, PtPIP2; 10 is the only PIP in which the Phe at $\mathrm{H} 2$ position is substituted by an Asn, making it more hydrophilic. (B) Residues forming the ar/R filter of PtSIPI;I are very hydrophobic. Even the normally conserved Arg at LE2 position is substituted by a hydrophobic Phe. This will be one of the most hydrophobic constrictions in known MIPs.

similar to the ar/R filter of PtNIP3;1 and PtNIP3;2 in which the positions of hydrophobic and Thr (or Ser) residues are interchanged in the positions $\mathrm{H} 2$ and $\mathrm{H} 5$. In the second group, the Ala at LE1 position of the first group is replaced by Val making it more hydrophobic than the first group. In the third group, hydrophobic residues Val and Ile occupy three out of four positions (H2, H5 and LE1) with the conserved Arg at LE2 retained. This results in a highly hydrophobic environment at the pore constriction (Figure 4A) and it is somewhat similar to PtSIP1;1. The last group with one protein (PtXIP1;4) has ar/R tetrad similar to some of the NIP members of rice and maize (OsNIP2;1, OsNIP2;2, OsNIP3;2, OsNIP4;1, ZmNIP2;1 and $\mathrm{ZmNIP} 2 ; 2$ ). Small residues Ala/Thr are observed in three out of four positions making the constriction larger. In general, dicot XIP members from groups II and III significantly deviate from other subfamilies of PtMIPs and display more hydrophobic character at the ar/R selectivity filter compared to other PtMIPs.

Comparison of ar/R filters in moss XIPs (Table 6) indicates that all three of them have different signatures and hence each one can be considered as a separate group. PpXIP1;1 has a signature similar to a TIP protein from rice and maize (OsTIP4;2 and ZmTIP4;3). Similarly, ar/R tetrad of PpXIP1;2 has resemblance to another TIP protein from rice and maize (OsTIP5;1 and ZmTIP5;1). Interestingly, these two ar/R motifs are not found in Arabidopsis. The third XIP from moss has a Tyr at $\mathrm{H} 2$ position and Tyr residue has not been observed as part of the ar/ $\mathrm{R}$ signature in any of the 160 plant MIPs analyzed from the four plant species. The ar/R filters of all three moss XIPs are more hydrophilic than their counterparts in dicot plants.

The only example from the protozoa has bulky residues in all four positions that form the ar/R filter. Danielson and Johanson [24] have observed that this non-plant sequence from amoeba has some of the sequence characteristics such as NPA boxes and ar/R filter different from other XIPs.

Majority of fungi XIP sequences ( 7 out of 9 forming group I) has ar/R tetrad in which the $\mathrm{H} 2$ position is occupied by Asn (Table 6). Small residues are found in H5 and LE1 
Table 6: Ar/R filters of 35 XIPs from different organism groups

\begin{tabular}{|c|c|c|c|c|c|}
\hline Group $^{\mathbf{a}}$ & XIP Members & $\mathrm{H} 2$ & H5 & LEI & LE2 \\
\hline & Dicot Plants & & & & \\
\hline I & $\begin{array}{l}\text { PtXIPI;I, PtXIPI;2, PtXIPI;3 } \\
\text { CcXIPI;I, CcXIPI;2, CcXIPI;3, CcXIPI;4 } \\
\text { CsXIPI;I, CsXIPI;2 } \\
\text { VvXIPI;I, VvXIPI;2 } \\
\text { LsXIPI;I } \\
\text { SIXIPI;I }\end{array}$ & $\mathrm{V} / \mathrm{I}$ & $\mathrm{T}$ & A & $\mathrm{R}$ \\
\hline II & $\begin{array}{l}\text { PtXIPI;5 } \\
\text { GrXIPI;I } \\
\text { RcXIPI;I, RcXIP2;I, RcXIP2;2 } \\
\text { AfXIPI;I }\end{array}$ & $\mathrm{V} / \mathrm{I}$ & $T / S$ & V & $\mathrm{R}$ \\
\hline III & PtXIP2;I, RcXIP2;3 & $\mathrm{V} / \mathrm{I}$ & I & $\mathrm{V}$ & $\mathrm{R}$ \\
\hline \multirow[t]{2}{*}{ IV } & $\mathrm{PtXIPI;4}$ & $A$ & $\mathrm{~T}$ & $A$ & $\mathrm{R}$ \\
\hline & Moss & & & & \\
\hline 1 & PpXIPI;I & Q & $A$ & $A$ & $\mathrm{R}$ \\
\hline II & $\mathrm{PpXIPI;2}$ & Q & 1 & $\mathrm{~T}$ & $\mathrm{R}$ \\
\hline \multirow[t]{2}{*}{ III } & SmXIPI;I & $Y$ & S & $A$ & $\mathrm{R}$ \\
\hline & Protozoa & & & & \\
\hline \multirow[t]{2}{*}{ I } & DdXIP & $\mathrm{H}$ & 1 & $\mathrm{~F}$ & $\mathrm{R}$ \\
\hline & Fungi & & & & \\
\hline I & $\begin{array}{l}\text { F-FoXIP, F-GmXIP, } \\
\text { F-AtXIP, F-TaXIP, } \\
\text { F-TrXIP, F-TvXIPI, } \\
\text { F-PmXIP }\end{array}$ & $\mathrm{N}$ & $\mathrm{A} / \mathrm{S} / \mathrm{G}$ & A & $R / K$ \\
\hline ॥ & F-TsXIP & $\mathrm{N}$ & $S$ & L & $\mathrm{R}$ \\
\hline III & F-TvXIP2 & $A$ & $\mathrm{G}$ & $\mathrm{F}$ & $\mathrm{R}$ \\
\hline
\end{tabular}

aStructural subclasses for each organism group are defined based on the residues forming ar/R tetrad.

positions and the highly conserved Arg is observed in LE2 (F-TaXIP has a Lys residue in this position; Figure 4B). This signature is very different from that of dicot plant XIPs which are more hydrophobic. However, the group I fungi XIPs shows striking similarity with the ar/R filter of a moss XIP (PpXIP1;1) which in turn is similar to some of the rice and maize TIPs. Asn in $\mathrm{H} 2$ position is replaced by Gln in PpXIP1;1 and other features of ar/R filter are retained. Similarly, F-TsXIP from group II of fungi MIPs has ar/R signature similar to that of PpXIP1;2. The weakly polar and hydrophobic residues at H5 and LE1 positions are interchanged in the moss XIP. The XIP forming the third group in fungi (F-TvXIP2) is the only example that shows some similarity to group I dicot XIPs. One hydrophobic, two small/weakly polar residues with the conserved Arg at LE2 is the characteristic of ar/R motif in this group which is also shared by some members of Populus NIPs (PtNIP3;1 and PtNIP3;2).

In summary, PtMIPs that do not belong to XIP subfamily have ar/R selectivity filter similar to those found in Arabidopsis, rice and maize. Residues forming ar/ $\mathrm{R}$ tetrad in fourteen dicot XIP sequences are found to be similar to the NIP sequences from the Populus, rice and maize. The ar/R selectivity filters of the remaining eight dicot XIPs are more hydrophobic in nature and lack counterparts in other subfamilies of plants considered in this study. On the other hand, the moss and fungi XIPs have ar/R constriction that are more hydrophilic and similar to rice and maize TIPs. The analysis of ar/R selectivity filters based on homology modeling shows clear distinction between dicot XIPs and moss/fungi XIPs.

\section{Comparison of loops in XIPs and other MIP subfamily members}

Although transmembrane segments in aquaporin give structural scaffold and define the channel environment, loops connecting the TM helices also have significant role in the function of the channel such as gating [49] and could possibly be involved in selectivity also $[58,81]$. Among the five loops (A to E), the high conservation of residues observed in loops $\mathrm{B}$ and $\mathrm{E}$ are due to these loops possessing the NPA signature motif and their residues defining the channel interior and selectivity filter. The loop A, connecting $\mathrm{H} 1$ and $\mathrm{H} 2$, was used to discriminate the groups within Populus PIP family [73]. Loops C and D have been implicated in solute selectivity [58] and gating [49] respectively. Hence features observed in these loops 

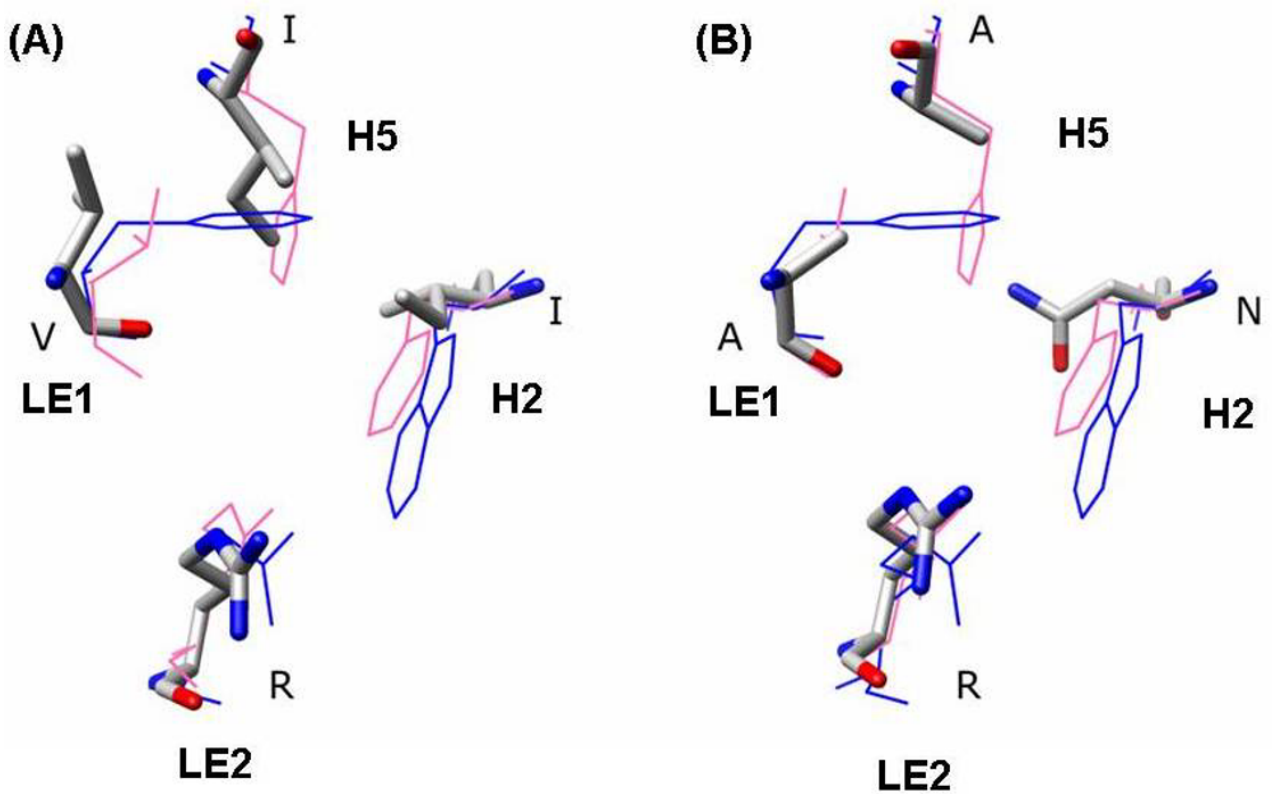

\section{Figure 4}

Ar/R selectivity filters of PtXIP2; I and F-FoXIP. Ar/R selectivity filters of two XIPs, one from a dicot plant (PtXIP2;I) and the other from fungi (F-FoXIP). XIP models were first individually superposed on the experimentally determined structures of GlpF and SoPIP2; I as described in Figure 3. Residues of XIP models are shown in stick representation with nitrogen and oxygen atoms displayed in blue and red respectively. For other details, see the caption of Figure 3. (A) Ar/R selectivity filter of PtXIP2; I has three hydrophobic residues and is likely to transport a more hydrophobic solute. (B) The presence of Asn and Arg along with two small residues makes the ar/R selectivity filter of F-FoXIP more hydrophilic and result in a wider constriction. Such XIPs are likely to transport bulkier hydrophilic solutes.

could be an important factor in giving rise to (i) different MIP subgroups, (ii) determining the nature of solute that is transported and (iii) functioning of the channel itself. We specifically focused on the loops C and D to find out whether they could be used to discriminate PtXIPs from the other Populus subfamily members. We also analyzed dicot XIPs and fungi/moss XIPs separately. We first used structure-based sequence alignment to segregate sequences in the loop regions and then used T-COFFEE [79] method to align only the part belonging to the respective loop regions from all MIP sequences and also independently from the subfamilies.

\section{Loop C}

Among the four known plant MIP subfamilies, the lengths of loop C in PIPs and a subgroup of SIPs (SIP2s) are the largest (> 20 residues) and the smallest (14 residues) respectively (Table 7 ). Exceptions are observed in few members. For example, ZmTIP5; 1 has 29 residues. However, the same analysis for XIP members show some interesting features. In general, the length of loop $\mathrm{C}$ can be used to distinguish the dicot and moss XIPs from other plant MIP subfamilies. All 18 dicot XIPs belonging to the first subgroup (XIP1s) are observed to have much longer
$\mathrm{C}$ loop with 33 residues (Figure 5). The length of the same loop in XIP2 members is shorter by 8 residues, but still 5 residues longer than plant PIPs. Surprisingly, the loop C of all moss XIPs are similar to the dicot XIP1s and all are having loop $\mathrm{C}$ with more than 30 residues. Fungi XIPs, on the other hand, has much shorter loop $\mathrm{C}$ among all XIPs and its length is comparable to that of plant PIPs with 20 residues (Figure 5).

Analysis of loop C residues indicates that some MIP families are enriched with Gly residues in this loop. All XIPs have at least three Gly residues and dicot XIPs have more Gly residues than any other MIPs (Table 7). Twenty out of 22 dicot XIPs have at least five Gly residues in loop C (Figure 5). Similarly, loop C in 52 out of 54 PIPs contains at least four Gly residues (Additional file 6). However, TIPs and NIPs possess less number of Gly in loop $C$ than their counterparts in PIPs and XIPs, although some exceptions are seen. For example, OsNIP1;2 and OsNIP1;5 have respectively 9 and 7 Gly residues in loop C. SIPs have the least number of Gly ( 2 or 1 ) in this loop. The longer loop and larger number of Gly residues indicate that the loop $\mathrm{C}$ in dicot XIPs is much more flexible than other MIP members. 
Table 7: Analysis of loops C and D in MIPs from different organism groups

\begin{tabular}{|c|c|c|c|c|c|}
\hline MIP Subfamily & No. of sequences & Length of the loop C & Loops with $\geq 3$ Gly & Length of loop D & $\begin{array}{l}\text { No. of basic residues } \\
\text { in loop } D\end{array}$ \\
\hline Dicot XIPIs & 18 & 33 & All dicot, XIPs & $15-16$ & $\geq 3$ (19 out of 22$)$ \\
\hline Dicot XIP2s & 4 & 25 & & & \\
\hline Moss XIPs & 3 & $31-34$ & All & $12-16$ & I (2 out of 3$)$ \\
\hline Fungi XIPs & 9 & $20--23$ & All & 16 & 2 (8 out of 9 ) \\
\hline All PIPs & 54 & $21--22$ & 53 out of 54 & $13--14$ & 4 (53 out of 54$)$ \\
\hline All TIPs & 49 & 17 & 40 out of 49 & $9--11$ & 2 (4I out of 49 ) \\
\hline All NIPs & 37 & $16-24$ & 12 out of 37 & $8--9$ & I (32 out of 37 ) \\
\hline All SIPs & 14 & $14-19$ & None & $8--9$ & $2(10$ out of 14$)$ \\
\hline $\begin{array}{l}\text { Human glycerol } \\
\text { transporting AQPs }\end{array}$ & 4 & 35 & All & 10 & $\begin{array}{l}0 \text { (2 out of } 4) \\
2(2 \text { out of } 4)\end{array}$ \\
\hline Other Human AQPs ${ }^{b}$ & 9 & $20-23$ & AQPI, AQP4 & $5--9$ & 2 basic ( 7 out of 9 ) \\
\hline
\end{tabular}

${ }^{a} A Q P 3, A Q P 7, A Q P 9, A Q P I 0$ are the human AQP homologs that transport glycerol.

bAQP0, AQPI, AQP2, AQP4, AQP5, AQP6, AQP8, AQPII, AQPI2

When we analyzed the loop $\mathrm{C}$ of human counterparts, four out of thirteen human aquaporins (AQP3, AQP7, $\mathrm{AQP9}$ and $\mathrm{AQP10}$ ) contain 35 residues in loop $\mathrm{C}$ and all four also possess at least 3 Gly residues (Table 7). These human MIP homologs are known to be glycerol transporters, a feature also shared by the prototype glycerol transporter, the bacterial GlpF. GlpF with 39 residues in loop C is one of the longest known in aquaporin family. Most of the other human aquaporins have loop C with 20 to 23 residues, shorter by more than 10 residues compared to their glycerol-transporting counterparts. Although, it is tempting to correlate the length of loop $\mathrm{C}$ with the glycerol transporting property, several plant NIPs are known to transport glycerol [65] and they have much shorter loop $\mathrm{C}$ and their length is only half of what is observed in dicot XIPs and GlpF. However, the fact that the loop C residues have a role to play in the selectivity of solute transport has support from experimental studies (see Discussion).

\section{Loop D}

The crystal structure of plant plasma membrane aquaporin clearly demonstrates the involvement of loop D in gating of the channel [49]. Loop D is, in general, shorter than loop C. Among the four major non-XIP subfamilies, PIPs have longer D loop with 13 to 14 residues (Additional file 7). D loops in SIPs are the shortest with 8 to 9 residues (Table 7 ). There are some exceptions like AtPIP1;4 and AtNIP1; 1 that have more than 20 residues in loop D. Analysis of loop D sequences in XIPs indicates that all of them have slightly longer loop D (15 to 16 residues) compared to that of plant MIPs from other subfamily members (Figure 6).

Computational studies on a mammalian AQP1 have indicated that the basic residues in loop D could be significant in cation transport in the central channel formed by the tetramer [82]. We have examined the occurrence of charged residues in loop D of all plant MIP families (Table 7). In general, loop D in dicot XIPs is more basic, having at least three basic residues compared to their counterparts in moss and fungi (Figure 6). The loop D of all the fungi XIPs is rich in proline residues and no proline is observed in the same loop in majority of dicot XIPs. Among non-XIP members, PIPs have four basic residues compared to two or less in TIPs, NIPs and SIPs (Additional file 7). Similarly, two out of four glycerol-transporting human AQPs have less number of basic residues than other human homologs. This analysis indicates that the possible influence of loop $\mathrm{D}$ in gating of the central channel could be different in different species.

\section{Group conservation of residues at the helix-helix interface} Analysis of high-resolution crystal structures of MIP homologs showed that small and weakly polar residues (Ala, Gly, Ser, Thr and Cys) occur at the helix-helix interface of transmembrane helix bundle $[21,54,83]$. Structure-based sequence alignment of 105 MIP sequences from Arabidopsis, rice and maize indicated that these residues are conserved as a group at the helix-helix interface 


\begin{abstract}
Dicot XIP1s
PtXIP $1 ; 1$

PtXIP $1 ; 2$

PtXIP $1 ; 3$

PtXIP $1 ; 4$

PtXIP $1 ; 5$

AfXIP $1 ; 1$

CCXIP $1 ; 1$

CCXIP $1 ; 2$

CCXIP $1 ; 3$

CCXIP $1 ; 4$

CSXIP $1 ; 1$

CSXIP $1 ; 2$

GrXIP $1 ; 1$

LSXIP $1 ; 1$

$\operatorname{RCXIP} 1 ; 1$

SIXIP $1 ; 1$

VVXIP $1 ; 1$

VVXIP $1 ; 2$

Moss XIPs

PpXIP $1 ; 1$

PPXIP $1 ; 2$

SmXIP $1 ; 1$

Dicot XIP2s

PtXIP 2; 1

$\operatorname{RCXIP} 2 ; 1$

$\operatorname{RCXIP} 2 ; 2$

$\operatorname{RCXIP} 2 ; 3$

Fungi XIPs

F-AtXIP

F-FOXIP

$\mathrm{F}-\mathrm{GmXIP}$

$\mathrm{F}-\mathrm{PmXIP}$

$\mathrm{F}-\mathrm{T}$ aXIP

F-TrXIP

F-TsXIP

F-TVXIP $1 ; 1$

F-TVXIP $1 ; 2$

Protozoan XIP

DdXIP

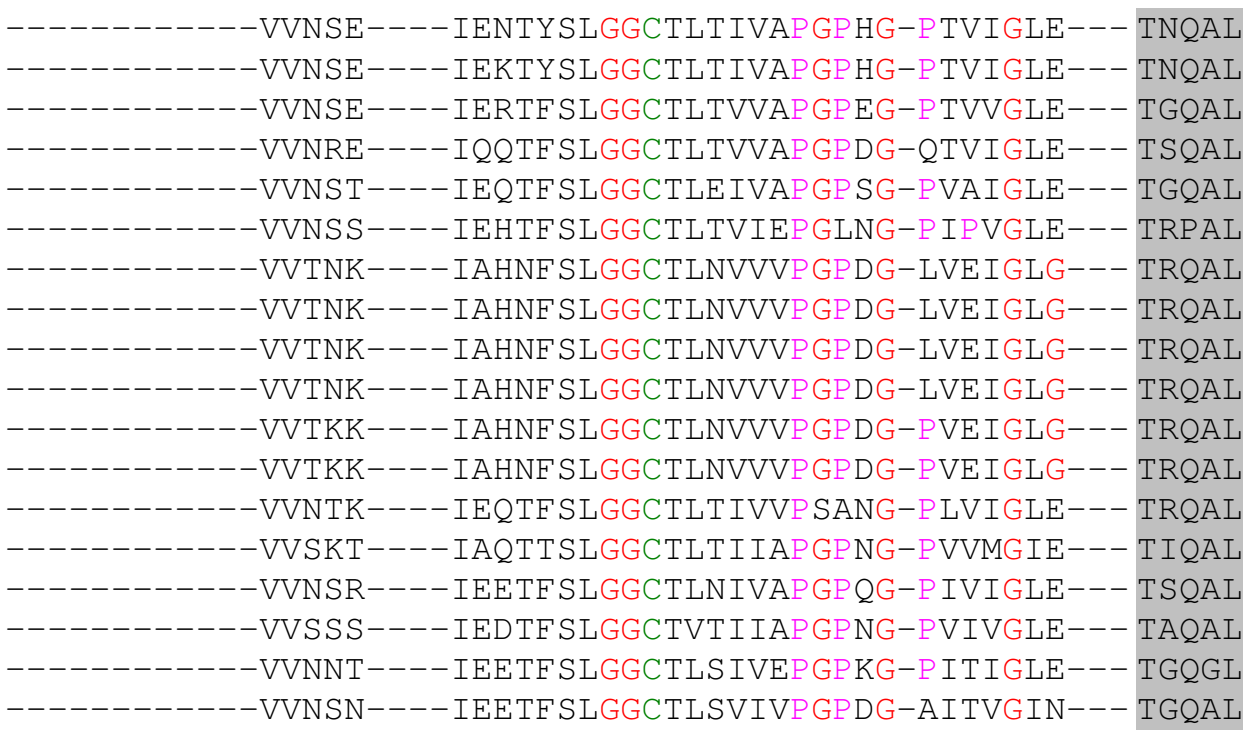

FMAKI ------------VVGNA----LATQYNLGGCYLQSRVSATSG---MMGLG--- TGRAL

LGMWA ------------LTTHE----MRREYSLGGCLLQKLP--VEG--TDLGLSTLS NKQGL

LAAKQ ------------VVARD----FVEKNSLAGCLLGEVVRTSRGLEWPEGLT--- PRAGL
\end{abstract}

\title{
Figure 5
}

Alignment of loop $\mathbf{C}$ residues of XIPs. The sequence regions containing loop $\mathrm{C}$ are aligned for all XIPs. Residues forming the last turn of $\mathrm{H} 3$ and the first turn of $\mathrm{H} 4$ are shown in gray background. All Gly and Pro residues are displayed in red and pink color respectively. The conserved Cys which is part of the 'GGC' motif is shown in green.

at 17 positions in MIP proteins [21]. High abundance of such residues helps to mediate helix-helix interactions and close packing of helices [84]. In this study, we have analyzed the group conservation at those 17 positions by considering 55 Popular MIPs and all the XIPs using structure-based sequence alignment. Our results show that in Populus MIPs also small and weakly polar residues are group conserved at the helix-helix interface (Table 8). As observed in the other three plant species, PtPIPs have the highest conservation in which all 17 positions are 100\% group conserved (Additional file 8). This is followed by PtTIPs (82 - 100\%) and PtNIPs (91-100\%). Group conservation at helix-helix interface is in general high in PtSIPs and PtXIPs, although some positions are poorly 


\section{Dicot XIP1s}

$\begin{array}{ll}\text { PtXIP } 1 ; 1 & \text { SVWMA } \\ \text { PtXIP } 1 ; 2 & \text { SVWMA } \\ \text { PtXIP } 1 ; 3 & \text { SVWMA } \\ \text { PtXIP } 1 ; 4 & \text { SVWMA } \\ \text { PtXIP } 1 ; 5 & \text { SIYIA } \\ \text { A } 1 \text { XIP } 1 ; 1 & \text { SVSIA } \\ \text { CCXIP } 1 ; 1 & \text { SVWMA } \\ \text { CCXIP } 1 ; 2 & \text { SVWMA } \\ \text { CCXIP } 1 ; 3 & \text { SVWMA } \\ \text { CCXIP } 1 ; 4 & \text { SVWMA } \\ \text { CSXIP } 1 ; 1 & \text { SVWMA } \\ \text { CSXIP } 1 ; 2 & \text { SVWMA } \\ \text { GrXIP } 1 ; 1 & \text { SIWIA } \\ \text { LSXIP } 1 ; 1 & \text { SIWLA } \\ \text { RCXIP } 1 ; 1 & \text { SIWLA } \\ \text { SIXIP } 1 ; 1 & \text { SIWMA } \\ \text { VVXIP } 1 ; 1 & \text { SVWIA } \\ \text { VVXIP } 1 ; 2 & \text { SVWIG } \\ \text { Dicot XIP2S } & \end{array}$

\section{Dicot XIP2s}

PtXIP $2 ; 1$

$\operatorname{RCXIP} 2 ; 1$

$\operatorname{RCXIP} 2 ; 2$

$\operatorname{RCXIP} 2 ; 3$

Moss XIPs

\begin{abstract}
PPXIP $1 ; 1$
PPXIP $1 ; 2$

SmXIP $1 ; 1$
\end{abstract}

\section{Fungi XIPs}

F-AtXIP
F-FOXIP
F-GmXIP
F-PmXIP
F-TaXIP
F-TrXIP
F-TsXIP
F-TVXIP $1 ; 1$
F-TVXIP $1 ; 2$

Protozoan XIP

\section{DdXIP}

\section{GVTVA \\ AMTI I \\ AMTIV \\ AMT I I}

SYSVA

VYGIG

AYSTL

AFGVA

AFGVG

AFGVG

AFGVA

AFGVG

SFGVG

AFGVA

SFGVG

SFGVG
SFGT
FDHRQAQGIGRVGVF I

FDHRQAQGIGRVGVF I

FDHRQAKGLGRVNVLI

FDQRQAKALGRVNVF I

FDRRQAIALGRVVFCS

FDERQIKAR-PVTVFT

FDSRQAKALGRVTVCI

FDSRQAKALGRVTVCI

FDSRQAKALGRVTVCI

FDSRQAKALGRVTVCI

FDSRQAKALGRVTVCI

FDSRQAKALGRVTVCI

FDKRQAKHLGRVVVCS

YDDRQAKSLGIVIVFS

FDKRQAKLLGQVIVCS

YDHRQAKALGHVTVLS

FDERQAKVHGAVVVCS

FDDRQAKALGLVLVCS

FDKRRCKELGLQMVCG

LDKKKCQELGLTMVCV

LDKKKCQDLGLTMVCI

LDKQKCMDLGLTTVCV

LDPPRLPRTGYTLAPF

FDSRNVVVT-FLI---

LEP GNFRQTGP ILAAL

LDPRQAKIFGHATSPW

LDPRQAKI I GPALGPF

LDPRQAKI I GPALGPF

LNPRQERIYGPALAPW

LDPRQAALF GP RMGPA

LDPRQAALF GP RMGP L

LNPRQERIFGPALAPW

LDPRQAALF GP RMGP L

LDPRQRSVFGPALGP I
IGGIV
IGGIV
IVGIV
IIGIV
IIGLV
IIGIL
VIGTV
VIGTV
VIGTV
VIGTV
VIGTV
VIGTV
IIGVV
IIGVV
IIGLV
IVGLV
IIGTV
IIGAV

ILAGA

IISGA

IISGA

IISGI

MIGGI

SSPFI

SIGGA

FVGIV

MVGLS

MVGLS

LVGLA

LVGAS

LVGAS

LVGLT

LVGAS

FVGLV

Figure 6

Alignment of loop D residues of XIPs. Multiple sequence alignment of residues forming loop D is shown for all XIPs. Residues forming the last turn of $\mathrm{H} 4$ and the first turn of $\mathrm{H} 5$ are shown in gray background. All basic (Arg, Lys and His) and acidic (Asp and Glu) residues are displayed in red and blue colors respectively. ClustalW (version I.82) was used to perform the multiple sequence alignment of both loops. 
Table 8: Group conservation of small and weakly polar residues at the helix-helix interface of PtMIPs and all XIPs

\begin{tabular}{|c|c|c|c|}
\hline Residue $^{\mathrm{a}}$ & All PtMIPsb, c & Dicot XIPsc & Fungi $X I P s^{c}$ \\
\hline T48 & $\mathrm{T}(9 \mathrm{I}), \mathrm{S}, \mathrm{A}(95)$ & $\mathrm{T}(86), \mathrm{S}(100)$ & $\mathrm{T}(44), \mathrm{S}(67)$ \\
\hline T55 & $\mathrm{G}(45), \mathrm{T}(25), \mathrm{S}, \mathrm{A}(\mathbf{8 0})$ & $\mathrm{T}(5)$ & $S(33), T, A(67)$ \\
\hline A78 & $\mathrm{A}(69), \mathrm{S}, \mathrm{C}, \mathrm{T}(9 \mathrm{I})$ & $S(73), A(82)$ & $\mathrm{G}(100)(100)$ \\
\hline G82 & $G(5 I), A(4 I)(9 I)$ & $A(73), S(77)$ & -- \\
\hline Al03 & $A(76), S, T(87)$ & $\mathrm{T}, \mathrm{A}(23)$ & $\mathrm{T}(44), \mathrm{A}(56)$ \\
\hline GI07 & $\mathrm{G}(58), \mathrm{A}(25), \mathrm{S}, \mathrm{T}(98)$ & $A(50), S(32)(82)$ & $A(78), S(100)$ \\
\hline G129 & $\mathrm{G}(87), \mathrm{A}(100)$ & $\mathrm{G}(100)(100)$ & $G(100)(100)$ \\
\hline $\mathrm{A} \mid 30$ & $S(56), A(33), G, T(100)$ & $A(50), G(45), S(100)$ & $\mathrm{G}(89), \mathrm{A}(100)$ \\
\hline G133 & $A(55), G(45)(100)$ & $G(91), A(100)$ & $A(89), G(100)$ \\
\hline TI72 & $\mathrm{T}(82), \mathrm{G}, \mathrm{S}, \mathrm{A}(100)$ & $T(55), S(27), A(100)$ & $\mathrm{S}(33), \mathrm{T}, \mathrm{A}, \mathrm{C}(89)$ \\
\hline SI8I & $\mathrm{A}(42), \mathrm{S}(27), \mathrm{G}, \mathrm{T}(84)$ & $\mathrm{T}, \mathrm{S}(23)$ & $G(100)(100)$ \\
\hline G203 & $\mathrm{G}(87), \mathrm{A}, \mathrm{S}(100)$ & $G(82), A(100)$ & $\mathrm{G}(100)(100)$ \\
\hline S226 & $\mathrm{S}(60), \mathrm{A}, \mathrm{C}, \mathrm{T}(93)$ & $C(100)(100)$ & $C(100)(100)$ \\
\hline G248 & $\mathrm{G}(73), A, C, S(98)$ & $\mathrm{G}(95)(95)$ & $G(44), A(33), S(100)$ \\
\hline $\mathrm{G} 252$ & $\mathrm{G}(87), \mathrm{A}(98)$ & $\mathrm{A}(77), \mathrm{S}, \mathrm{G}(91)$ & $A(56), G(78)$ \\
\hline A253 & $\mathrm{A}(60), \mathrm{G}, \mathrm{C}, \mathrm{S}, \mathrm{T}(98)$ & $C(82), S, G(95)$ & G(33), C, T, A, S (89) \\
\hline A256 & $A(7 I), G(87)$ & -- & -- \\
\hline
\end{tabular}

aResidue numbers correspond to that of spinach aquaporin SoPIP2; I (PDB ID: IZ98)

bGroup-based conservation is reported for all the five populus MIP subfamilies based on structure-based sequence alignment of 55 Populus MIPs cSmall and weakly polar interfacial residues in Populus MIP sequences and their conservation (if it exceeds $25 \%$ ) are given. If the conservation is less than $25 \%$, only the residues are given. For each position, group conservation of all the five residues (Gly, Ser, Thr, Ala and Cys) is shown in bold and italics.

conserved. The conservation of Ala 78, Gly 82 and Ser 181 (the numbering followed here is that of 1298 , the structure of SoPIP2;1) is below 50\% in PtSIPs. Similarly, the positions corresponding to Thr 55, Ala 103, Ser 181 and Ala 256 are either poorly conserved $(<25 \%)$ or not conserved at all in PtXIPs. It must be mentioned that the number of sequences considered for PtXIPs is only six, and analysis of all 22 dicot XIP sequences also gives rise to a similar observation (Table 8).

Analysis of 9 fungi XIPs indicates that the group conservation of small and weakly polar residues is $100 \%$ for 9 positions and is very high for another 5 positions. There are differences between dicot and fungi XIPs. For example, at position 181, although the group conservation is only $23 \%$ in dicot XIPs, Gly is $100 \%$ conserved in fungi XIPs. However, we observed the opposite at position 82 . In the dicot XIPs, the group conservation at this position is $77 \%$ while in the fungi XIPs, there is absolutely no conservation. Similarly, the position 55 is reasonably well conserved in fungi XIPs and there is poor conservation in dicot XIPs.

In the previous analysis, we have observed that subfamilies show strong preference for one or another amino acid at certain positions [21]. A similar trend is observed in Populus MIPs also. Notably, the position 226 is occupied by either Ser/Ala in PtPIPs, PtTIPs, PTNIPs and PtSIPs. In PtXIPs a strong preference for Cys is observed at that position (Additional file 8). Similarly, at position 253 $\mathrm{Ala} / \mathrm{Gly}$ is predominantly found in the four non-XIP sub- families and a preference for Cys is found in PtXIPs at the same position. This is also confirmed in the analysis of 35 XIPs and all of them have Cys at position 226. In position 253, only dicot XIPs shows a strong preference for Cys (Table 8).

\section{Gene Structure of MIPs}

Non-XIP Populus MIPs

The availability of three plant genomes, two dicotyledons and one monocotyledon, enabled us to analyze and compare the gene structures of MIP genes belonging to different subfamilies and different species. Recently, gene structures of MIPs from the avascular plant Physcomitrella patens have also been analyzed [24]. Although the exonintron organization of AtMIPs has been reported [18], comparison of MIP gene structures across the three plant species has not been carried out. We have compared the gene structures of PtMIPs with that of OsMIPs and AtMIPs. In general, they show that the number and positions of introns are unique and are conserved within each subfamily of a given species. However, major differences are observed when the subfamilies from dicots are compared with those from the monocot.

Comparison of members from PIP subfamily shows that the gene structures of majority of PtPIPs have three introns, similar to that of AtPIPs (Figure 7). However, only 3 out of 11 OsPIPs have the same organization. Eight OsPIPs have lost at least one intron (two of the OsPIPs belonging to the indica-cultivar group have been excluded from this analysis). OsPIP1;3 and OsPIP2;7 have only one 


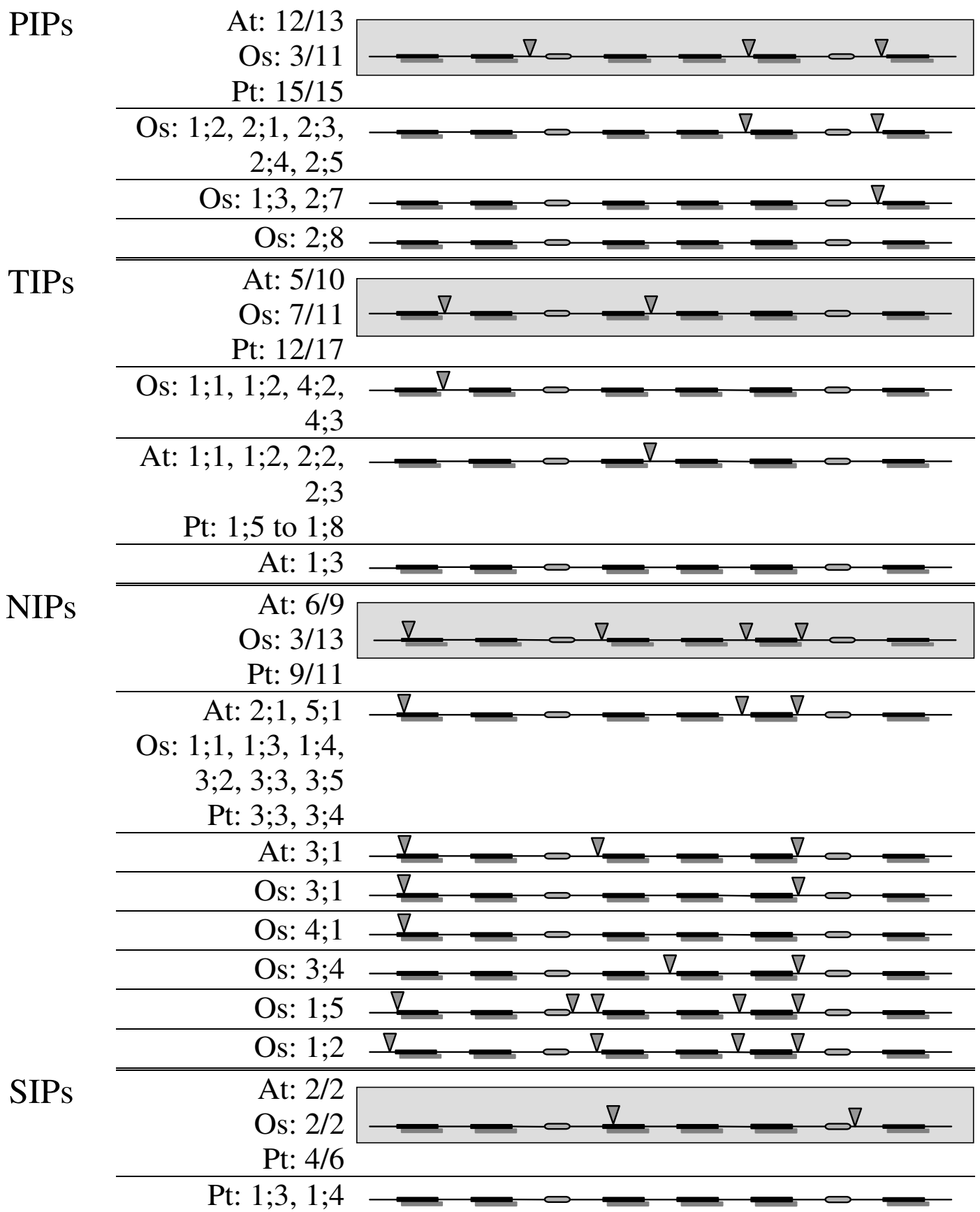

\section{Figure 7}

Gene structure of non-XIP MIPs from Populus, Arabidopsis and rice. Exon-intron organization of non-XIP MIP genes from Populus, Arabidopsis and rice is depicted for the PIP, TIP, NIP and SIP subfamilies. The exon-intron pattern observed in majority of MIPs within a subfamily is shown in gray background. In this case, only the number of MIPs having that pattern is indicated for each plant species. For example, "At: 12/13" indicates that 12 out of I 3 AtPIPs have the same gene structure. For those members with different exon-intron organization, the MIP name is explicitly given (example: Os:2.8 in PIP subfamily). The six TM regions are shown in black bars and the loops $B$ and $E$ are shown in oval shape. The intron positions are indicated by inverted triangle. 
intron and OsPIP2; 8 has no intron. In most of the OsPIPs, the intron between the helices $\mathrm{H} 2$ and $\mathrm{H} 3$ has been lost. A similar result is observed for NIP subfamily (Figure 7). Most of the PtNIPs have gene structures similar to that of AtNIPs. Four introns are observed in 9 out 11 PtNIPs. Gene structures of OsNIPs diverged from their counterparts in Arabidopsis and Populus. At least one intron is lost in nine out of 13 OsNIPs and they have three introns or less. In most of them, the intron between the TM helices $\mathrm{H} 2$ and $\mathrm{H} 3$ is lost as in OsPIPs. Members of SIP subfamily have two introns in Arabidopsis, rice and also most of the Populus SIPs. PtSIP1;3 and PtSIP1;4 have no introns. Most of the Populus (12 out of 17) and rice (7 out of 11) TIP members and half of AtTIPs (5 out of 10) have two introns. Four TIP members, each from rice, Arabidopsis and Populus have lost one intron. However, it must be pointed out that the intron lost in rice TIPs is not the same as that observed in the other two dicot plant TIPs.

The gene structures of the non-XIP MIPs in two dicot plants, Populus and Arabidopsis, are very similar (Figure 7). Both PIP and NIP subfamilies have three and four introns respectively in these two plants. The number and locations of introns in the PIP and NIP subfamilies of moss plant Physcomitrella patens [24] are the same as that observed in their counterparts in the two dicot plants. On the other hand, intron loss is observed in rice PIP and NIP subfamilies. This could be a general feature observed in dicot and monocot PIP and NIP subfamilies and such intron loss could have happened when monocots diverged from dicots.

\section{Populus XIPs versus moss/fungi XIPs}

The pattern of exon -introns in five out of six PtXIPs has already been reported and compared with that of two moss XIPs [24]. Two introns in the N-terminal region are observed in six out of seven XIPs. Due to the high degree of variation observed in the N-termini, no conclusion was reached regarding the conservation of intron positions between the moss plant and Populus. Since the fungi XIPs have been identified from their respective genome sequences, it is possible to derive the gene structure of these MIP sequences and compare them with that of Populus and Physcomitrella. It is interesting to note that in addition to the N-terminal intron, six out of nine fungi XIPs have at least one additional intron (Figure 8). In all six of them, an intron is present between helices $\mathrm{H} 5$ and H6. In three cases, additional introns are present between helices $\mathrm{H} 2$ and $\mathrm{H} 3$ and also between $\mathrm{H} 3$ and $\mathrm{H} 4$.

\section{Transcript abundance of non-PtXIPs and PtXIPs}

Expression levels of all Populus MIPs were analyzed using an Affymetrix microarray-based Poplar genome arrays [85] as described in the Methods section. We have reanalyzed the Populus transcript abundance data generated by Wilkins et al [85]. Transcript abundance of 50 out of 55 PtMIPs are available in the microarray dataset. There were no probe sets for two TIPs (PtTIP5;1 and PtTIP5;2) and three NIPs (PtNIP1;1, PtNIP1;2 and PtNIP1;5). Heatmap

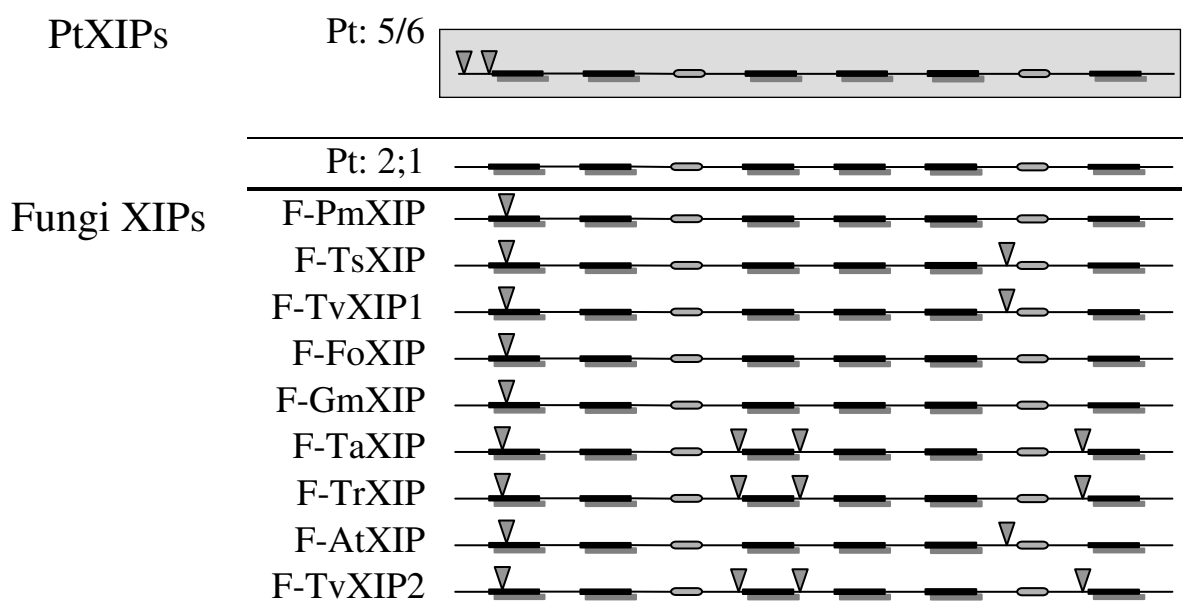

\section{Figure 8}

Exon-intron pattern observed in Populus and fungi XIPs. Exon-intron organization of XIP MIP genes from Populus and fungi is displayed. Five out of 6 PtXIPs have similar exon-intron organization and the pattern is shown in gray background. The sixth PtXIP (PtXIP2;I) has no introns and its gene structure is shown separately. The six TM regions are shown in black bars and the loops $B$ and $E$ are shown in oval shape. The intron positions are indicated by inverted triangle. For details about fungi XIPs, see Table 3. 
(Figure 9) is produced for the remaining Populus MIPs using the expression profiles obtained for nine different tissues (seedlings grown under three different light conditions, young and mature leaves, female and male catkins, roots and xylem). Probe sets were clustered using hierarchical clustering and the heatmap is displayed using this clustering based on the transcript abundance pattern using the program Heatplus [86]. Major PtMIPs that are expressed in xylem, a tissue responsible for the woody stem, are PtPIPs and PtTIPs. A similar result is observed in root tissues also. Maximum number of PtTIPs is expressed in seeds grown in different light conditions. PtNIPs and PtSIPs are the predominant members expressed in male and female catkins. No appreciable accumulation of transcripts in mature leaf and seedlings grown in continuous darkness is found for NIPs and SIPs. The same is true for PIPs in female catkins and seedlings grown in continuous darkness and then transferred to light for $3 \mathrm{hrs}$. PtXIPs are expressed in seven of the nine tissues studied. Only in xylem and female catkins, no member of XIPs is found to be expressed. Transcript abundance of two XIPs is found in male catkins, root and three tissues of seedlings grown in different light conditions. A single XIP is expressed in mature leaf (PtXIP1;5) and young leaf (PtXIP2;1).

\section{Discussion}

Due to whole-genome duplication events, the number of protein-coding genes in Populus is more than that observed in Arabidopsis [67]. In the present study, we have found 55 Populus MIP genes and this is much higher compared to the total of 35 Arabidopsis MIPs. Our studies show that Populus has $~ 1.6$ times MIP genes than those found in Arabidopsis. This agrees with the reported observation, based on comparative genomics studies, that for each Arabidopsis gene, 1.4 to 1.6 putative Populus homologs are found [67]. The number of MIPs from rice and maize is also found to be less than forty [19-21]. MIPs from these four plants have been compared. Phylogenetic analysis reveals that Populus MIPs can be divided into five subfamilies. The four known subfamilies PIPs, TIPs, NIPs and SIPs are present in all the four plant species considered in this study. Members of the fifth subfamily, XIPs, are uncharacterized and are absent in Arabidopsis, rice and maize. Recent studies have identified XIPs in the primitive plant Physcomitrella patens. TIPs and SIPs are present in larger number in Populus compared to the other three plants. While Populus has 17 TIPs and 6 SIPs, Arabidopsis, rice and maize each have only 10 to 11 TIPs and 2 to 3 SIPs. The higher number of MIPs found in Populus is mainly attributed to the presence of higher number of PtTIPs and PtSIPs in addition to the six PtXIPs.

\section{Non-XIP MIPs from eudicot genomes have similar ar/R filters and gene structures}

Homology modeling was used to analyze the aromatic/ arginine selectivity filters of plant MIPs. Ar/R tetrads from non-XIP PtMIPs were analyzed and compared with that of their counterparts from Arabidopsis, rice and maize. Ar/R filters of only three out of 49 non-XIP PtMIPs seem to be different from the other three plants. Although, the larger number of TIPs in Populus indicated the possible diversity in the solutes transported by this subfamily, analysis of ar/ $\mathrm{R}$ selectivity filters of all PtTIPs indicated otherwise. They are identical to AtTIPs and no member of PtTIP was found to have ar/R filter that can be described as novel. Similarly, nine out of 11 PtNIP members have counterparts in AtNIPs. One or two examples are found in NIP and SIP members where the ar/R filter is identical or similar to rice/maize members. The analysis of ar/R selectivity filters did not find any surprises and it shows that majority of non-XIP PtMIPs are similar to their counterparts in Arabidopsis.

The availability of two eudicot genomes (Populus and Arabidopsis) and one monocot genome (rice) helps us to analyze and compare the gene structures of plant MIPs. Differences observed in the pattern of exon - intron organization of MIPs from these three plant species can explain the evolution of eudicot MIP gene family and also the divergence of monocot MIPs from dicots. Intron loss is observed in majority of the OsPIPs and OsNIPs compared to the same subfamilies in Arabidopsis and in Populus. The loss of introns observed in OsPIPs and OsNIPs might have occurred independently during the evolution of rice to achieve genome slimming [87]. It is also tempting to speculate that the intron loss in rice might have happened during the divergence of monocotyledonous and dicotyledonous plants that occurred about 200 million years ago (Mya) [88]. However, such generalization is possible only after analyzing plant MIP gene structures from a large number of monocot and dicot plants. In this context, we would like to point out the recent work of Roy and Penny [89] who have observed a high degree of intron loss along a wide variety of eukaryotic lineages. They have also found that intron losses have outnumbered intron gains during the evolution of plants.

\section{XIPs in dicots and fungi differ in Ar/R selectivity filter, loop $C$ and gene structure}

Our TBLASTN search on plant EST databases and fungi genomic sequences identified additional XIPs from dicot plants and fungi. In total, we considered 35 XIPs from dicots, fungi and moss for characterizing this new subfamily. We analyzed several features including the nature of ar/R selectivity filters, loop lengths, conservation of residues at the helix-helix interface and gene structures and these features were compared between different species 

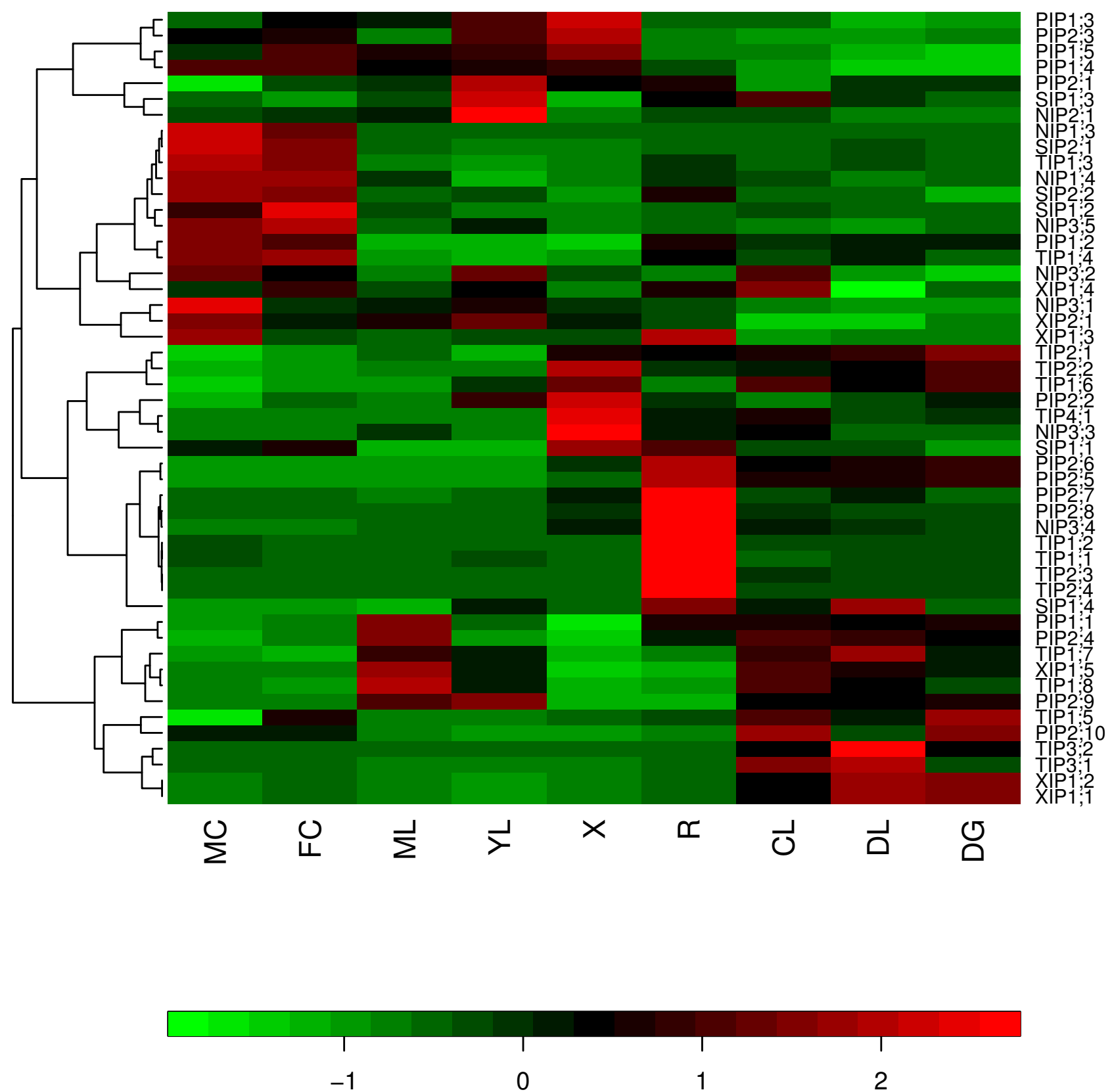

Figure 9

Relative transcript abundance profiles of Populus MIPs. A heat map showing the transcript abundance of MIPs from all Populus subfamilies [85] is displayed using the program "Heatplus" [86]. This heat map is produced using the expression data obtained from Populus eFP browser [103]. The transcript abundance levels for the Populus MIPs were clustered using hierarchical clustering based on Pearson correlation coefficients. Each row corresponds to the normalized expression profile of a particular gene and their names are shown. Data obtained for nine different tissues for each gene are represented in columns. Symbols in the map represent as follows: ML -- mature leaf; YL -- young leaf; R -- root; DG -- dark-grown seedlings, etiolated; DL -- dark grown seedling etiolated and then exposed to light for $3 \mathrm{hrs}$; CL -- continuous light-grown seedling; FC -- female catkins; MC -- male catkins; $X$-- xylem. The data is normalized for each gene (row-normalized). The relative transcript accumulation is represented in a color code with green and red showing respectively the lower and higher levels of transcript accumulation. 
groups within XIPs to understand the evolution of this uncharacterized subfamily. Comparison was also made between XIPs and other four subfamilies of Populus.

\section{Ar/R filters in XIPs are hydrophilic in moss/fungi and more hydrophobic in dicot plants}

Homology models of XIPs were analyzed and divided into structural subclasses based on the nature of residues that constitute the ar/R selectivity filters. The 22 dicot XIPs were divided into four structural subclasses. Fourteen of them from two groups are similar to the NIP subgroups from the four plants analyzed in this paper. Eight dicot XIPs from the remaining two groups have bulky hydrophobic residues occupying two/three of the four positions. Some SIPs from Populus, rice and maize have such arrangement although they lack the conserved Arg at LE2 position. All the three XIPs from moss and eight out of 9 fungi XIPs have hydrophilic residues occupying two out of four positions. Small residues are found in the remaining two positions of most of the fungi and all the moss XIPs. This arrangement is very similar to some of the rice and maize TIPs but it is not found in Populus and Arabidopsis. In general, the ar/R selectivity filters of fungi and moss are more hydrophilic than their dicot counterparts. This clearly indicates that the nature of solutes that are transported by dicot XIPs will be very different from their counterparts in fungi/moss.

Ar/R filters of some of the XIPs are presented in the recent work of Danielson and Johanson [24]. Two possibilities are given for the residue at $\mathrm{H} 5$ position in their work. In the present study, when the target sequence was aligned with the template sequences during homology modeling procedure, it resulted in aligning the conserved Gly in H5 and hence in our models, the residue at $\mathrm{H} 5$ position of ar/ $\mathrm{R}$ filters is the alternate residue reported in their paper [24]. This residue is Ser/Thr in most of the cases. Even if we consider the other possibility for $\mathrm{H} 5$ position (Val/Ile for dicot XIPs) as reported in [24], the ar/R filter of dicot XIPs will become even more hydrophobic compared to fungi/moss XIPs. There is also a disagreement in the ar/R tetrad reported for SmXIP1;1. Our model shows that the $\mathrm{H} 2$ position in this moss XIP is occupied by a Tyr residue, whereas Danielson and Johanson [24] have reported a Leu residue at this position. Our structure-based sequence alignment clearly shows that Tyr is more likely to occupy this position (data not shown) which also makes the ar/R filter more hydrophilic as observed in the other two moss XIPs (PpXIP1;1 and PpXIP1;2)

Can the length of loop $C$ be used as an indicator of XIP subfamilies? Although the significance of loops $\mathrm{B}$ and $\mathrm{E}$ is one of the most well established in aquaporin channel's function, recent crystal structures from the plant spinach [49] and the malarial parasite Plasmodium falciparum [58] have indicated the role of two other loops D and C in the channel's gating and selectivity. Loop $\mathrm{C}$ connects the two halves of the channel protein linking the transmembrane segments $\mathrm{H} 3$ and H4. The length of this loop from known crystal structures varies from 20 residues in water-transporting human AQP1 [52] to 39 residues in glyceroltransporting GlpF [53]. This loop tucks into the channel core towards the ar/R selectivity filter and comes in close contact with the Arg residue at LE2 position of ar/ $\mathrm{R}$ tetrad. The nature of residues in loop $\mathrm{C}$ is suggested to influence the solute molecules approaching the extracellular vestibule $[58,81]$. The length of loop $\mathrm{C}$ seems to be characteristic of different plant MIP subfamilies. Analysis of loop C in XIP members shows variation among the XIP subfamilies. Dicot XIP1s, moss XIPs, glycerol-specific GlpF and all four glycerol-transporting human AQP homologs have loop $\mathrm{C}$ that is more than 30 residues long. Dicot XIP2s and fungi XIPs have a smaller loop C with 20 to 25 residues as observed in other human AQP homlogs. Loop $\mathrm{C}$ in GlpF has been suggested to provide an attractive site for glycerol in the periplasmic vestibule [81]. Although, there seems to be a correlation between the nature of solute transport and the length of the loop $\mathrm{C}$, this relationship could not be very clearly established. For example, it appears that all glycerol-transporting MIPs will have a long loop $\mathrm{C}$ with $>30$ residues. However, several plant NIPs have been shown to transport glycerol [65] and some of them have much shorter loop $\mathrm{C}$ with less than 20 residues. Similarly, if we assume that all water-transporting channels have shorter loop $\mathrm{C}$ with $<25$ residues, then the moss XIPs with hydrophilic ar/R selectivity filter are likely to transport water along with other hydrophilic solutes with their $\mathrm{C}$ loop having more than 30 residues. A clearer picture is likely to emerge if we have functional data on more MIPs that can be directly linked with the length of loop C.

We have also recognized another interesting feature that some of the MIP families are enriched with Gly residues in loop C. Dicot XIPs and PIPs have at least five and four Gly residues respectively in loop $\mathrm{C}$. It could be that these Gly residues are present to impart flexibility to the loop or they could adopt conformations that are not allowed for other residues. We have examined the conformations of the three Gly residues that are part of the 'GGG' motif in both chains of spinach PIP structure (PDB ID: 1Z98; [49]). All three Gly residues have a positive $\phi$ value $(+66$ to $\left.+102^{\circ}\right)$ and a $\psi$ value close to zero $\left(-12\right.$ to $\left.+17^{\circ}\right)$ and this conformation is not accessible to other residues. Hence it is possible that Gly residues in the "GGGxN" and "GGC" motifs of PIPs and XIPs in loop C could play an important conformational role. 


\section{Intron loss is observed in moss and Populus XIPs}

Exon-intron pattern helps us to understand the evolution of XIP genes. Since all the Populus and fungi genes were identified from their respective genome sequences and the gene structure of Physcomittrella XIPs have been already reported [24], it was possible to compare the exon-intron organization of these 17 XIPs ( 6 from Populus, 9 from fungi and 2 from $P$. Patens). While six out of 9 fungi MIPs have at least two introns, a single intron at the $\mathrm{N}$-terminus is found in Populus and the moss XIPs. It appears that intron loss has occurred during the evolution when the moss plants diverged from fungi with moss XIPs having retained only the $\mathrm{N}$-terminal intron. When moss plants further diverged to dicotyledons, no introns were inserted in the coding region of XIPs. While the gene structures of moss and dicot XIPs are similar, the fungi with more introns have different pattern of exon-intron organization.

\section{Subfunctionalization of XIPs: Expression profiles of non-PtXIPs vs PtXIPs}

Members of Populus XIP family are expressed in seven out of nine tissues indicating that they don't show any tissuespecific transcript abundance. The fact that XIPs are not found in monocots and they are not particularly specific to any tissue indicates that the functions of XIP members might have been taken over by other MIP members during evolution. Clustering on the basis of transcript abundance pattern shows that PtXIP1;1 and PtXIP1;2 are grouped with PtTIP3;1 and PtTIP3;2. Analysis of ar/R selectivity filters of these members does indicate similar features. The positions of a bulky hydrophobic residue and a polar residue at $\mathrm{H} 2$ and $\mathrm{H} 5$ positions in PtXIP1;1 and PtXIP1;2 are exchanged in PtTIP3;1 and PtTIP3;2. Similarly, the transcript accumulation of PtXIP1;5 is most similar to PtTIP1;8 and both their ar/R tetrads have two bulky hydrophobic residues. PtXIP1;3 and PtXIP1;4 have closely related transcription abundance profiles with PtNIP3;1 and PtNIP3;2. Both the groups share two small residues in the ar/R selectivity filters. Thus analysis of Populus microarray data has indeed cast a light on likely members that could replace PtXIPs in monocots. It is possible that the XIP members became "functionally redundant" during evolution and the above TIP and NIP members could have substituted the functions of the redundant XIPs.

\section{Evolution of dicot XIPs}

Several reports, including fossil studies and molecular clock estimates have speculated the animal and plant evolutionary lines. Recent protein sequence analysis has estimated that major lineages of fungi were present more than 1000 million years ago and land plants appeared after 300 million years [90]. Analysis of MIPs from primitive organisms to higher animals will help to understand the evolution of these channel proteins and their trans- port mechanisms of diverse solutes at molecular level. Analysis of ar/R selectivity filters, loops and exon-intron organization of 34 XIPs from fungi, moss and dicot plants has given an idea about the evolution of this subfamily of aquaporins from fungi to higher plants (XIP from protozoa is not included in this Discussion). The hydrophilic ar/R selectivity filter in the fungi and moss XIPs indicates that these MIPs are likely to be involved in transport of hydrophilic solutes including water. The emergence of higher plants could possibly indicate more diversity in the solutes that are transported. The amino acids in the hydrophilic ar/R selectivity filters of fungi and moss XIPs were substituted by hydrophobic residues during the divergence of higher plants and this selectivity filter has become more hydrophobic in the dicot XIPs. As a result, the dicot XIPs are likely to be involved in solutes that are more hydrophobic than those transported by their counterparts in fungi and moss. With no XIP homolog found in monocots, at least the XIPs might have been replaced by some of the TIPs and NIPs with similar ar/R selectivity filters and transcription abundance profiles. The loop $\mathrm{C}$ in moss XIPs is longer than that of fungi XIPs and hence an insertion of more than 10 residues has occurred in the loop C of moss XIPs. While this length is retained in XIP1 group of dicot plants, the dicot XIP2s have loop $\mathrm{C}$ that is shorter by 8 residues. Hence, a deletion event seems to have occurred when dicot XIP2s evolved from moss or diverged from dicot XIP1s. As far as the loop D is concerned, dicot XIPs have more basic residues in loop D than their counterparts in fungi/moss and the only other subfamily with more number of basic residues in loop D is PIPs. As suggested for AQP1 [82], loop D in XIPs could be involved in activating the central tetrameric ion channel upon binding to some signaling molecule. Although evolution has made its mark in the selectivity filters and loops, the group conservation of small and weakly polar residues in the helix-helix interface of the $\alpha$-helical bundle observed in other MIP subfamilies is largely maintained in XIPs also. Analysis of exon-intron pattern suggests that intron loss has occurred in XIP genes when fungi diverged from the lineage leading to primitive and higher plants. In summary, during divergence from fungi and moss, the ar/ $\mathrm{R}$ selectivity filters of dicot XIPs has become more hydrophobic, loop $\mathrm{C}$ has become longer in a subgroup of dicot XIPs and loop D has become more basic. Moreover, analysis of gene structure indicates that moss/Populus XIPs lost introns when they evolved from fungi. The evolutionary features observed for dicot XIPs are summarized in Figure 10. Some of the observations made in this study will be strengthened as more genome sequences are available for different kingdoms and we will have a better understanding of the evolution of MIPs at molecular level. 


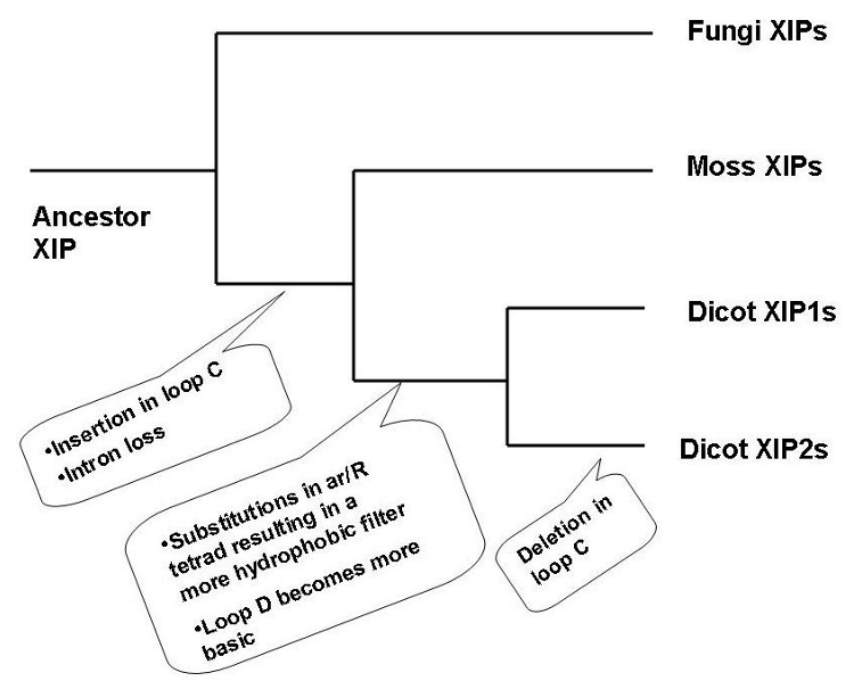

Figure 10

Evolution of dicot XIPs. A likely scenario for the evolution of dicot XIPs. The evolutionary events are indicated at the point where the XIPs diverged. Dicot XIPs evolved from fungi and moss through substitutions at ar/R selectivity filter, insertion/deletion of loop $C$ and loss of an intron. However, small and weakly polar residues occurring at the helix-helix interface are highly group-conserved.

\section{Conclusion}

We have analyzed 55 Populus MIP sequences and compared them with those from Arabidopsis, rice and maize. In addition to the four known MIP subfamilies, Populus has an additional uncharacterized XIP subfamily. The nonXIP Populus members are similar to their counterparts in the other three plants. The ar/R selectivity filters of majority of PtMIPs and the characteristics of loops $\mathrm{C}$ and $\mathrm{D}$ are similar to AtMIPs, OsMIPs and ZmMIPs. As far as the gene structures are concerned, the number and positions of introns are conserved within each subfamily of a given species. However, the inter-species comparison indicates that PIPs and NIPs of monocots lost introns when they diverged from eudicots.

We have also characterized 35 XIPs belonging to four different taxonomic groups. Our results show that in comparison to a hydrophilic selectivity filters in fungi and moss XIPs, substitutions in ar/R selectivity filters led to a more hydrophobic constriction in dicot XIPs. A longer loop $\mathrm{C}$ due to insertion is observed when moss and a subgroup of dicot XIPs evolved from fungi. When fungi XIPs diverged, intron loss is observed in moss and dicot XIPs. Analysis of microarray data indicates that Populus XIPs are expressed in almost all the tissues studied and they don't show any unique tissue-specific expression. While substitutions in ar/R tetrad and insertion/deletion events in loops reflect the divergence of these channel proteins, a high conservation of small and weakly polar residues as a group at the helix-helix interface is observed in all MIP subfamilies. Presumably, such group conservation helps to maintain the structural integrity of this channel protein during evolution. Our results indicate that in comparison to their counterparts in fungi and moss, dicot XIPs are likely to transport more hydrophobic solutes. Loop $\mathrm{C}$ in dicot XIPs in general and XIP1 subgroup in particular will have a potential influence in the selectivity of the solutes.

\section{Methods \\ Identification of Populus MIP genes}

The genome sequence of Populus trichocarpa female individual "Nisqually 1 " clone [67] was searched for MIP genes using TBLASTN $[76,80]$. The whole genome shotgun sequence (WGS) of Populus available at the National Center for Biotechnology Information (NCBI) [75] was used for this purpose with a rice MIP protein sequence (OsPIP $2 ; 1)$ as a query sequence. The hits thus obtained were subjected to phylogenetic clustering (see below). One representative sequence from each cluster was chosen as query sequence to identify additional and more distantly related Populus MIP homologs. Regions in Populus WGS contigs containing MIP genes were used to find out the gene structure using the program GeneMark.hmm ES$3.0[77,78]$. This version of GeneMark program is based on self-training algorithm for prediction of genes from novel eukaryotic genomes. There is significant similarity between Populus and Arabidopsis at the genome level and also the relative frequency of protein domains [67]. Between these two organisms, there is similarity in the codon usage also [70]. Hence, for gene prediction in Populus, Arabidopsis was chosen as a model organism in GeneMark. The predicted MIP genes were further compared with the Populus EST sequences available at NCBI and also the Populus EST database "PopulusDB" [70,72]. The KOG (euKaryotic Orthologous Groups) browser in Joint Genome Institute (JGI) [91] was also looked for Populus MIP genes.

The program T-COFFEE [79] was used to perform multiple sequence alignment on MIP protein sequences which was then used to generate phyologenetic tree. Three different methods were used to construct the evolutionary relationship among the sequences. They include neighborjoining method as implemented in Clustal (Version 1.82) [92] and heuristic searches using distance and parsimony methods as available in PAUP* version 4.0.0d55 in GCG package (Wisconsin Package version 10.3, Accelrys Inc., San Diego, California). The stability of branches in the resulting trees was confirmed by 100 bootstrap trails for all the three methods. The program TreeView [93] was used to display the trees. 


\section{Homology modeling of plant MIPs}

Three-dimensional models of Populus MIPs and other MIP proteins were built using the same protocol described in our earlier studies [21] to build models of Arabidopsis, rice and maize MIPs and it is briefly described below. Modeling procedure consisted of two stages. In the first stage, the software package MODELLER [94,95] was used to construct homology models of plant MIPs. In the second stage, the program SCWRL3 [96] was used to predict the side-chain conformation. MODELLER derives a set of spatial restraints on the structure of the target sequence using its alignment with the sequence of template structure(s). The resulting model is derived by optimizing the violations of all spatial restraints. The quality of the model is usually improved by considering more than one structure as template. In this study, we used the structures of mammalian AQP1 [52], bacterial GlpF [53] and archael AQPM [55] simultaneously as templates in their comparative modeling procedure. Their unique PDB IDs are $\underline{1 \mathrm{~J}} 4 \mathrm{~N}$, $\underline{1 F X} 8$ and 2 F2B respectively. All are high resolution structures (resolution 1.7 to $2.2 \AA$ ) and show different water permeabilities [96]. Using the program 'GAP' available in GCG package and the scoring matrix BLOSUM62, we found the pairwise sequence alignment of all Populus MIPs with the three template sequences. The average pairwise sequence identities between Populus MIPs and the three templates range from 21 to $45 \%$. Template sequences were first aligned based on a multiple structural superposition and then the target sequence was aligned. The target-template alignment was manually checked to find out if there is any gap in the middle of a transmembrane helical region or in the conserved loops B or E. If necessary, this alignment was manually refined. We have also analyzed more than 800 MIP sequences from diverse organisms (Gupta and Sankararamakrishnan, Manuscript in preparation) and found that at least one residue in each transmembrane segment (E17, G59, Q103, E144, G175 and $\mathrm{P} 218$ respectively in TM1 to TM6; $1 \mathrm{~J} 4 \mathrm{~N}$ numbering) is very highly conserved. We have exploited this information during the alignment of target and template sequences and hence there is less ambiguity in transmembrane segments. The models were built with the resultant target-template alignment using a 'very fast' simulated annealing optimization protocol. Ten models were built for each target sequence and the one with the lowest MODELLER objective function was selected. The refinement of loops and the side-chain conformations of nonconserved residues were carried out by MODELLER's loop optimization procedure and the graph theory-based SCWRL3 [96] method respectively. Finally, the model was minimized using GROMACS $[97,98]$ and its stereochemical quality was evaluated using PROCHECK [99]. Pore diameter profile of the model along its pore axis was calculated using the program HOLE [100] as described in Bansal and Sankararamakrishnan [21].

\section{Populus microarray analysis}

The transcript abundance of all Populus MIPs was analyzed using PopGenExpress, an Affymetrix microarray-based resource for poplar transcriptome analysis [85]. Expression data was obtained in biological triplicate RNA samples extracted in nine tissues by Malcolm Campbell and coworkers [85] and we have reanalyzed this transcript abundance data to find out whether there is any pattern of transcript accumulation in Populus MIP members. The microarray data corresponding to these experiments can be accessed in the NCBI's GEO database [101] (accession number: GES13990). Probe sets corresponding to the putative Populus MIPs were identified using Probe Match, a tool available as part of the NetAffx Analysis Center [102]. The identified probe sets were then used in the Populus electronic fluorescent pictograph browser (Poplar eFP browser) [103] to find out the transcript abundance levels. For genes with more than one probe sets, the median of expression values were considered. When two genes have the same probe set, then they are considered to have same level of transcript accumulation. The probe sets were then clustered using hierarchical clustering based on Pearson coefficients and the program Heatplus available in Bioconductor package [86] was used to display the expression pattern.

\section{Authors' contributions}

RS conceived the project. RS and ABG designed the work. $\mathrm{ABG}$ carried out the work. RS and ABG wrote the manuscript. Both authors approved the final version of the manuscript.

\section{Additional material}

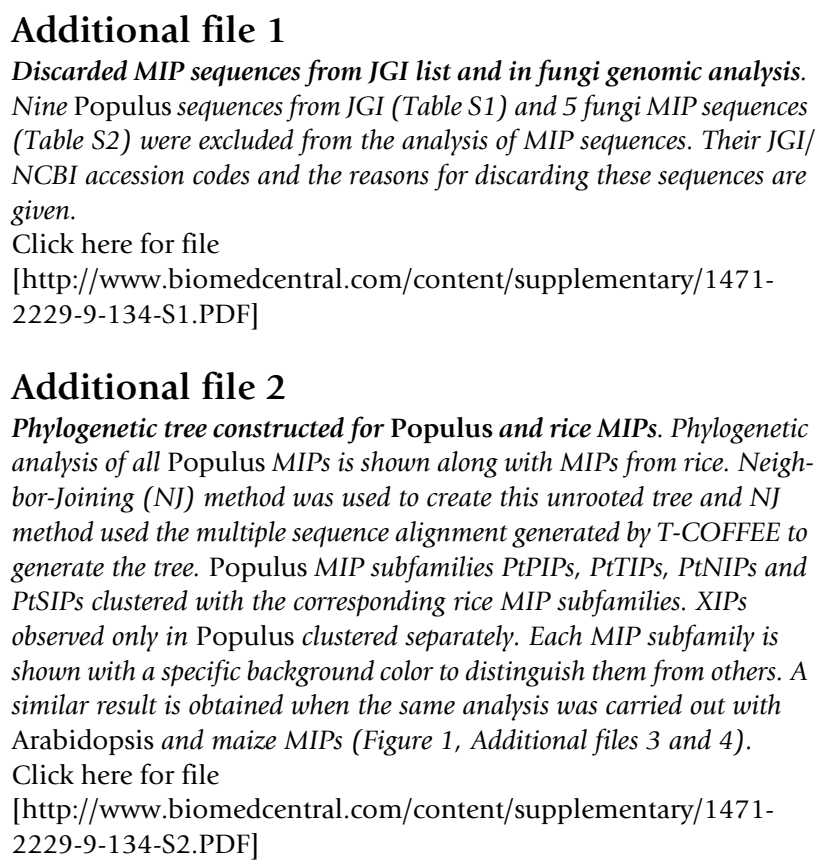

Additional file 2

Phylogenetic tree constructed for Populus and rice MIPs. Phylogenetic analysis of all Populus MIPs is shown along with MIPs from rice. Neighbor-Joining (NJ) method was used to create this unrooted tree and NJ method used the multiple sequence alignment generated by T-COFFEE to generate the tree. Populus MIP subfamilies PtPIPs, PtTIPs, PtNIPs and PtSIPs clustered with the corresponding rice MIP subfamilies. XIPs observed only in Populus clustered separately. Each MIP subfamily is shown with a specific background color to distinguish them from others. A similar result is obtained when the same analysis was carried out with Arabidopsis and maize MIPs (Figure 1, Additional files 3 and 4). Click here for file

[http://www.biomedcentral.com/content/supplementary/14712229-9-134-S2.PDF] 


\section{Additional file 3}

Phylogenetic tree constructed for Populus and maize MIPs. Phylogenetic analysis of all Populus MIPs is shown along with MIPs from maize. Neighbor-Joining (NJ) method was used to create this unrooted tree and NJ method used the multiple sequence alignment generated by T-COFFEE to generate the tree. Populus MIP subfamilies PtPIPs, PtTIPs, PtNIPs and PtSIPs clustered with the corresponding maize MIP subfamilies. XIPs observed only in Populus clustered separately. Each MIP subfamily is shown with a specific background color to distinguish them from others. A similar result is obtained when the same analysis was carried out with Arabidopsis and rice MIPs (Figure 1, Additional files 2 and 4). Click here for file [http://www.biomedcentral.com/content/supplementary/14712229-9-134-S3.PDF]

\section{Additional file 4}

Phylogenetic tree constructed for all four plant MIPs. Phylogenetic analysis of all Populus MIPs is shown along with MIPs from the other three plants Arabidopsis, rice and maize. Neighbor-Joining (NJ) method was used to create this rooted tree and NJ method used the multiple sequence alignment generated by T-COFFEE to generate the tree. Populus MIP subfamilies PtPIPs, PtTIPs, PtNIPs and PtSIPs clustered with the corresponding MIP subfamilies from the other three plants. XIPs observed only in Populus clustered separately. Each MIP subfamily is marked separately. A similar result is obtained when the same analysis was carried out on Populus MIPs paired individually with Arabidopsis, rice and maize MIPs (Figure 1, Additional files 2 and 3). Numbers above branches indicate bootstrap support. Populus aquaporins belonging to different subfamilies are shown in different colors.

Click here for file

[http://www.biomedcentral.com/content/supplementary/14712229-9-134-S4.PDF]

\section{Additional file 5}

Phylogenetic analysis of Populus MIPs and all XIPs. Phylogenetic analysis of all Populus MIPs is shown along with XIPs from the other dicot plants, fungi, moss and protozoa. Neighbor-Joining (NJ) method was used to create this rooted tree and NJ method used the multiple sequence alignment generated by T-COFFEE to generate the tree. Members of Populus XIP subfamily clustered with other XIPs and this group is separate from PtPIPs, PtTIPs, PtNIPs and PtSIPs. Background of nonXIP subfamilies are shown in different colors to distinguish from each other and from XIPs.

Click here for file

[http://www.biomedcentral.com/content/supplementary/14712229-9-134-S5.PDF]

\section{Additional file 6}

Sequence alignment of loop $C$ residues of all non-XIP plant MIPs. The sequence regions containing loop $C$ are aligned for all non-XIP plant MIPs from Arabidopsis, Populus, rice and maize. Residues forming the last turn of $\mathrm{H} 3$ and the first turn of $\mathrm{H} 4$ are shown in gray background. All Gly and Pro residues are displayed in red and pink color respectively. The conserved residues within each subgroup are shown in green color.

Click here for file

[http://www.biomedcentral.com/content/supplementary/14712229-9-134-S6.PDF]

\section{Additional file 7}

Sequence alignment of loop D residues of all non-XIP plant MIPs. Multiple sequence alignment of loop D residues of all non-XIP plant MIPs. Residues forming the last turn of $\mathrm{H} 4$ and first turn of $\mathrm{H} 5$ are displayed in gray background. Acidic (Asp and Glu) and basic (Arg, Lys and His) residues are shown in blue and red respectively.

Click here for file

[http://www.biomedcentral.com/content/supplementary/14712229-9-134-S7.PDF]

\section{Additional file 8}

Group conservation of small and weakly polar conserved interfacial residues in Populus MIPs. Conservation of small and weakly polar residues (Gly, Ala, Ser, Thr, Cys) as a group is reported for all Populus MIPs and also individually for the subfamily members. Conservation was found out for 17 positions using structure-based sequence alignment of MIP sequences. The residues at these 17 positions occur at the helix-helix interface of the transmembrane helix bundle.

Click here for file

[http://www.biomedcentral.com/content/supplementary/14712229-9-134-S8.PDF]

\section{Acknowledgements}

We are extremely grateful to Vivek Modi for his timely help in Populus microraray data analysis. This research is partially supported by a grant from the Department of Biotechnology, Government of India (No. BT/ HRD/34/17/2008). ABG thanks Council of Scientific and Industrial Research (CSIR), India for a senior research fellowship. RS is a Joy Gill Chair Professor at IIT-Kanpur. We would like to thank the three anonymous reviewers for their helpful comments and suggestions. We thank all members of our laboratory for useful discussions.

\section{References}

I. Zhou Y, Setz N, Niemietz C, Qu H, Offler CE, Tyerman SD, Patrick $\mathrm{JW}$ : Aquaporins and unloading of phloem-imported water in coats of developing bean seeds. Plant Cell Environ 2007, 30:1566-1577.

2. Siefritz F, Tyree MT, Lovisolo C, Schubert A, Kaldenhoff R: PIP I plasma membrane aquaporins in tobacco: From cellular effects to function in plants. Plant Cell 2002, 14:869-876.

3. Maurel C: Plant aquaporins: Novel functions and regulation properties. FEBS Lett 2007, 58 I:2227-2236.

4. Bots $M$, Vergeldt F, Wolters-Arts $M$, Weterings $K$, van As $H$, Mariani C: Aquaporins of the PIP2 class are required for efficient anther dehiscence in tobacco. Plant Physiol 2005, 137:1049-1056.

5. Kaldenhoff $R$, Fischer M: Functional aquaporin diversity in plants. Biochim Biophys Acta 2006, I758: I I 34-I I4I.

6. Higuchi $T$, Suga $S$, Tsuchiya $T$, Hisada $H$, Morishima $S$, Okada $Y$, Maeshima M: Molecular cloning, water channel activity and tissue sepcific expression of two isoforms of radish vacuolar aquaporin. Plant Cell Physiol 1998, 39:905-913.

7. Kjellbom $P$, Larsson $C$, Johansson I, Karlsson $M$, Johanson $U$ : Aquaporin and water homeostasis in plants. Trends Plant $\mathrm{Sci}$ 1999, 4:308-314.

8. Gao YP, Young L, Bonham-Smith P, Gusta LV: Characterization and expression of plasma and tonoplast membrane aquaporins in primed seed of Brassica napus during germination under stress conditions. Plant Mol Biol 1999, 40:635-644.

9. Kaldenhoff R, Ribas-Carbo M, Sans JF, Lovisolo C, Heckwolf M, Uehlein N: Aquaporins and plant water balance. Plant Cell Environ 2008, $31: 658-666$.

10. Uehlein N, Kaldenhoff R: Aquaporins and plant leaf movements. Annals Botany 2008, I0I:I-4. 
II. Lian HL, Yu X, Ye Q, Ding XS, Kitagawa Y, Kwak SS, Su WA, Tang ZC: The role of aquaporin RWC3 in drought avoidance in rice. Plant Cell Physiol 2004, 45:48I-489.

12. Peng YH, Lin WL, Cai WM, Arora R: Overexpression of a Panax ginseng tonoplast aquaporin alters salt tolerance, drought tolerance and cold acclimation ability in transgenic Arabidopsis plants. Planta 2007, 226:729-740.

13. Katsuhara M, Koshio K, Shibasaka M, Hayashi Y, Hayakawa T, Kasamo $\mathrm{K}$ : Over-expression of a barley aquaporin increased the shoot/root ratio and raised salt sensitivity in transgenic rice plants. Plant Cell Physiol 2003, 44: I 378-I383.

14. Mut P, Bustamante C, Martinez G, Alleva K, Sutka M, Civello M, Amodeo $G$ : A fruit-specific plasma membrane aquaporin subtype PIP I; I is regulated during strawberry (Fragaria $x$ ananassa) fruit ripening. Physiol Plantarum 2008, I32:538-55I.

15. Agre P, Preston GM, Smith BL, Jung JS, Raina S, Moon C, Guggino WB, Nielsen S: Aquaporin Chip - The archetypal molecular water channel. Am J Physiol 1993, 265:F463-F476.

16. Ishibashi K, Sasaki S, Fushimi K, Uchida S, Kuwahara M, Saito H, Furukawa T, Nakajima K, Yamaguchi Y, Gojobori T, et al.: Molecular cloning and expression of a member of the aquaporin family with permeability to glycerol and urea in addition to water expressed at the basolateral membrane of kidney collecting duct cells. Proc Natl Acad Sci USA 1994, 91:6269-6273.

17. Maurel C, Reizer J, Schroeder JI, Chrispeels MJ, Saier MH: Functional characterization of the Escherichia coli glycerol facilitator, GIpF, in Xenopus oocytes. J Biol Chem 1994, 269:। I869-I|872

18. Johanson U, Karlsson M, Johansson I, Gustavsson S, Sjovall S, Fraysse L, Weig AR, Kjellbom P: The complete set of genes encoding major intrinsic proteins in Arabidopsis provides a framework for a new nomenclature for major intrinsic proteins in plants. Plant Physiol 200I, I 26: I358-1369.

19. Chaumont F, Barrieu F, Wojcik E, Chrispeels MJ, Jung R: Aquaporins constitute a large and highly divergent protein family in maize. Plant Physiol 200I, I 25:1206-1215.

20. Sakurai J, Ishikawa F, Yamaguchi T, Uemura M, Maeshima M: Identification of 33 rice aquaporin genes and analysis of their expression and function. Plant Cell Physiol 2005, 46: I 568-I 577.

21. Bansal A, Sankararamakrishnan R: Homology modeling of major intrinsic proteins in rice, maize and Arabidopsis: comparative analysis of transmembrane helix association and aromatic/arginine selectivity filters. BMC Struct Biol 2007, 7:27.

22. Johanson U, Gustavsson S: $\mathbf{A}$ new subfamily of major intrinsic proteins in plants. Mol Biol Evol 2002, | 9:456-46।.

23. Gustavsson S, Lebrun A-S, Norden K, Chaumont F, Johanson U: A novel plant major intrinsic protein in Physcomitrella patens most similar to bacterial glycerol channels. Plant Physiol 2005 , I 39:287-295.

24. Danielson JAH, Johanson U: Unexpected complexity of the aquaporin gene family in the moss Physcomitrella patens. BMC Plant Biol 2008, 8:45

25. Zelazny E, Borst JW, Muylaert M, Batoko H, Hemminga MA, Chaumont $\mathrm{F}$ : FRET imaging in living maize cells reveals that plasma membrane aquaporins interact to regulate their subcellular localization. Proc Natl Acad Sci USA 2007 I04: | 2359-12364.

26. Ma JF, Tamai K, Yamaji N, Mitani N, Konishi S, Katsuhara M, Ishiguro M, Murata Y, Yano M: A silicon transporter in rice. Nature 2006, 440:688-69l.

27. Takano J, Wada M, Ludewig U, Schaaf G, von Wiren N, Fujiwara T: The Arabidopsis major intrinsic protein NIP5; I is essentia for efficient boron uptake and plant development under boron limitation. Plant Cell 2006, I 8:1498-I 509.

28. Liu LH, Ludewig U, Gassert B, Frommer WB, von Wiren N: Urea transport by nitorgen-regulated tonoplast intrinsic proteins in Arabidopsis. Plant Physiol 2003, 133: I 220-1228.

29. Maeshima M, Ishikawa F: ER membrane aquaporins in plants. Pflugers Arch Eur J Physiol 2008, 456:709-716.

30. Maurel C, Verdoucq L, Luu D-T, Santoni V: Plant aquaporins: Membrane channels with multiple integrated functions. Annu Rev Plant Biol 2008, 59:595-624.

31. Maurel C: Aquaporins and water permeability of plant membranes. Annu Rev Plant Physiol Plant Mol Biol 1997, 48:399-429.

32. Dean RM, Rivers RL, Zeidel ML, Roberts DM: Purification and functional reconstitution of soybean nodulin 26. An aquaporin with water and glycerol transport properties. Biochemistry 1999, 38:347-353.

33. Biela A, Grote K, Otto B, Hoth S, Hedrich R, Kaldenhoff R: The Nicotiana tabacum plasma membrane aquaporin NtAQPI is mercury-insensitive and permeable for glycerol. Plant J 1999 , I 8:565-570.

34. Wu B, Beitz E: Aquaporins with selectivity for unconventional permeants. Cell Mol Life Sci 2007, 64:24I3-242I.

35. Gaspar M, Bousser A, Sissoeff I, Roche O, Hoarau J, Mahe A: Cloning and characterization of ZmPIP I-5b, an aquaporin transporting water and urea. Plant Sci 2003, I65:2I-3I.

36. Kojima S, Bohner A, von Wiren N: Molecular mechanisms of urea transport in plants. J Memb Biol 2006, 2 I 2:83-9I.

37. Gerbeau P, Guclu J, Ripoche P, Maurel C: Aquaporin Nt-TIPa can account for the high permeability of tobacco cell vacuolar membrane to small neutral solutes. Plant J 1999, 1 8:577-587.

38. Choi WG, Roberts DM: Arabidopsis NIP2; I, a major intrinsic protein transporter of lactic acid induced by anoxic stress. J Biol Chem 2007, 282:24209-24218.

39. Dordas C, Brown PH: Evidence for channel mediated transport of boric acid in squash (Cucurbita pepo). Plant and Soil 200I, 235:95-103.

40. Bienert GP, Schussler MD, Jahn TP: Metalloids: essential, beneficial or toxic? Major intrinsic proteins sort it out. Trends Biochem Sci 2008, 33:20-26.

4I. Bienert GP, Thorsen M, Schuessler MD, Nilsson HR, Wagner A Tamas MJ, Jahn TP: A subgroup of plant aquaporins facilitate the bidirectional diffusion of $\mathrm{As}(\mathrm{OH}) 3$ and $\mathrm{Sb}(\mathrm{OH}) 3$ across membranes. BMC Biol 2008, 6:26.

42. Katsuhara M, Hanba YT: Barley plasma membrane intrinsic proteins (PIP aquaporins) as water and CO2 transporters. Pflugers Arch Eur J Physiol 2008, 456:687-69I.

43. Bienert GP, Moller ALB, Kristiansen KA, Schulz A, Moller IM, Schjoerring JK, Jahn TP: Specific aquaporins facilitate the diffusion of hydrogen peroxide across membranes. J Biol Chem 2007, 282: I 183-1192.

44. Bertl A, Kaldenhoff $\mathrm{R}$ : Function of a separate $\mathbf{N H}_{3}$-pore in aquaporin TIP2;2 from wheat. FEBS Lett 2007, 58 I:54 I 3-54I7.

45. Jahn TP, Moller ALB, Zeuthen T, Holm LM, Klaerke DA, Mohshin B, Kuhlbrandt W, Schjoerring JK: Aquaporin homologues in plants and mammals transport ammonia. FEBS Lett 2004, 574:3I-36.

46. Santoni V, Verdoucq L, Sommerer N, Vinh J, Pflieger D, Maurel C: Methylation of aquaporins in plant plasma membrane. Biochem J 2006, 400: I89-197.

47. Wallace IS, Choi WG, Roberts DM: The structure, function and regulation of the nodulin 26-like protein family of plant aquaglyceroporins. Biochim Biophys Acta 2006, I 758: I I 65-I I 75.

48. Daniels MJ, Yeager M: Phosphorylation of aquaporin PvTIP3; I defined by mass sepctrometry and molecular modeling. Biochemistry 2005, 44: |4443-|4454.

49. Tornroth-Horsefield S, Wang Y, Hedfalk K, Johanson U, Karlsson M, Tajkhorshid E, Neutze R, Kjellbom P: Structural mechanism of plant aquaporin gating. Nature 2006, 439:688-694.

50. Vera-Estrella R, Barkla BJ, Bohnert HJ, Pantoja O: Novel regulation of aquaporins during osmatic stress. Plant Physiol 2004 I35:2318-2329.

5I. Prak S, Hem S, Boudet J, Viennois G, Sommerer N, Rossignol M, Maurel C, Santoni V: Multiple phosphorylations in the C-terminal tail of plant plasma membrane aquaporins. Mol Cell Proteomics 2008, 7:1019-1030.

52. Sui H, Han B-G, Lee JK, Walian P, Jap BK: Structural basis of water-specific transport through the AQPI water channel. Nature 200I, 4I 4:872-878.

53. Fu D, Libson A, Miercke LJW, Weitzman C, Nollert P, Krucinski J Stroud RM: Structure of a glycerol-conducting channel and the basis for its selectivity. Science 2000, 290:48I-486.

54. Savage DF, Egea PF, Robles-Colmenares Y, O'Connell ID III, Stroud RM: Architecture and selectivity in aquaporins: 2.5 A structure of aquaporin Z. PLOS Biol 2003, I:334-340

55. Lee JK, Kozono D, Remis J, Kitagawa Y, Agre P, Stroud RM: Structural basis for conductance by the archaeal aquaporin AqpM at I.68 A. Proc Natl Acad Sci USA 2005, I02:18932-I8937.

56. Gonen T, Sliz P, Kistler J, Cheng Y, Walz T: Aquaporin-0 membrane junctions reveal the structure of a closed water pore. Nature 2004, 429:193-197. 
57. Harries WEC, Akhavan D, Miercke LJW, Khademi S, Stroud RM: The channel architecture of aquaporin 0 at a 2.2-A resolution. Proc Natl Acad Sci USA 2004, I 0 I: | 4045- | 4050.

58. Newby ZER, O'Connell ID III, Robles-Colmenares Y, Khademi S, Miercke LJW, Stroud RM: Crystal structure of the aquaglyceroporin PfAQP from the malarial parasite Plasmodium falciparum. Nature Struct Mol Biol 2008, I 5:619-625.

59. Hub JS, de Groot BL: Does CO2 permeate through aquaporinI? Biophys J 2006, 9 I:842-848.

60. de Groot BL, Grubmuller $\mathrm{H}$ : The dynamics and energetics of water permeation and proton exclusion in aquaporins. Curr Opin Struct Biol 2005, 15:176-183.

61. de Groot BL, Grubmuller H: Water permeation across biological membranes: Mechanism and dynamics of aquaporin-I and GIpF. Science 200I, 294:2353-2357.

62. Tajkhorshid E, Nollert P, Jensen MO, Miercke LJW, O'Connell JD III, Stroud RM, Schulten K: Control of the selectivity of the aquaporin water channel family by global orientational tuning. Science 2002, 296:525-530.

63. Jensen MO, Park S, Tajkhorshid E, Schulten K: Energetics of glycerol conduction through aquaglyceroporin GlpF. Proc Natl Acad Sci USA 2002, 99:673।-6736.

64. Beitz E, Wu B, Holm LM, Schultz JE, Zeuthen T: Point mutations in the aromatic/arginine region in aquaporin I allow passage of urea, glycerol, ammonia and protons. Proc Natl Acad Sci USA 2006, I 03:269-274.

65. Wallace IS, Roberts DM: Distinct transport selectivity of two structural subclasses of the nodulin-like intrinsic protein family of plant aquaglyceroporin channels. Biochemistry 2005, 44:16826-16834

66. Wallace IS, Roberts DM: Homology modeling of representative subfamilies of Arabidopsis major intrinsic proteins: Classification based on the Aromatic/Arginine selectivity filter. Plant Physiol 2004, I 35:1059-1068.

67. Tuskan GA, DiFazio S, Jansson S, Bohlmann J, Grigoriev I, Hellsten U, Putnam N, Ralph S, Rombauts S, Salamov A, et al:: The genome of black cottonwood, Populus trichocarpa (Torr. \& Gray). Science 2006, 3 13:1596-1604.

68. Jansson S, Douglas C): Populus : A model system for plant biology. Annu Rev Plant Biol 2007, 58:435-458.

69. Brunner AM, Busov VB, Strauss SH: Poplar genome sequence: functional genomics in an ecologically dominant plant species. Trends Plant Sci 2004, 9:49-56.

70. Sterky F, Bhalerao RR, Unneberg P, Segerman B, Nilsson P, Brunner AM, Charbonnel-Campaa L, Lindvall J], Tandre K, Strauss SH, et al.: A Populus EST resource for plant functional genomics. Proc Natl Acad Sci USA 2004, I 0 I:1395 I-13956.

7I. Sjodin A, Bylesjo M, Skogstrom O, Eriksson D, Nilsson P, Ryden P, Jansson S, Karlsson J: UPSC-BASE - Populus transcriptomics online. Plant J 2006, 48:806-8I7.

72. PopulusDB [http://www.populus.db.umu.se/]

73. Marjanovic Z, Uehlein N, Kaldenhoff R, Zwiazek JJ, Weib M, Hampp $\mathrm{R}$, Nehls U: Aquaporins in poplar: What a difference symbiont makes. Planta 2005, 222:258-268.

74. Kohler A, Delaruelle C, Martin D, Encelot N, Martin F: The poplar root transcriptome: analysis of 7000 expressed sequence tags. FEBS Lett 2003, 542:37-4I.

75. NCBI [http://www.ncbi.nlm.nih.gov/]

76. TBLASTN [http://www.ncbi.nlm.nih.gov/genome/seg/BlastGen/ BlastGen.cgi?taxid $=3694$ ]

77. Lomsadze A, Ter-Hovhannisyan V, Chernoff Y, Borodovsky M: Gene identification in novel eukaryotic genomes by self-training algorithm. Nucleic Acids Res 2005, 33:6494-6506.

78. GeneMark [http://exon.gatech.edu/GeneMark/]

79. Notredame C, Higgins DG, Heringa J: T-Coffee: A novel method for fast and accurate multiple sequence alignment. J Mol Biol 2000, 302:205-217.

80. Altschul SF, Madden TL, Schaffer AA, Zhang J, Zhang Z, Miller W, Lipman D]: Gapped BLAST and PSI-BLAST: a new generation of protein database search programs. Nucleic Acids Res 1997, 25:3389-3402

8I. Wang Y, Schulten K, Tajkhorshid E: What makes an aquaporin channel a glycerol channel? A comparative study of AqpZ and GIpF. Structure 2005, I3: | 107-1 | | 8.
82. Yu J, Yool AJ, Schulten K, Tajkhorshid E: Mechanism of gating and ion conductivity of a possible tetrameric pore in aquaporinI. Structure 2006, | 4: |4| |-|423.

83. Senes A, Ubarretxena-Belandia I, Engelman DM: The $\mathbf{C} \alpha-\mathbf{H}$... hydrogen bond: A determinant of stability and specificity in transmembrane helix interactions. Proc Natl Acad Sci USA 200 I, 98:9056-906I.

84. Eilers $M$, Patel $A B$, Liu W, Smith SO: Comparison of helix interactions in membrane and soluble $\alpha$-bundle proteins. Biophys J 2002, 82:2720-2736

85. Wilkins O, Nahal H, Foong J, Provart NJ, Campbell MM: Expansion and diversification of the Populus R2R3-MYB family of transcription factors. Plant Physiol 2009, I 49:98I-993.

86. Heatplus [http://www.bioconductor.org/packages/bioc/html/Heat plus.html]

87. Petrov DA, Lozovskaya ER, Hartl DL: High intrinsic rate of DNA loss in Drosophila. Nature 1996, 384:346-349.

88. Laroche J, Li P, Bousquet J: Mitochondrial DNA and monocotdicot divergence time. Mol Biol Evol I995, I2: I I5I-I I 56.

89. Roy SW, Penny D: Patterns of intron loss and gain in plants: Intron-loss-dominated evolution and genome-wide comparison of O. Sativa and A. thaliana. Mol Biol Evol 2007, 24: I7I-I8I.

90. Heckman DS, Geiser DM, Eidell BR, Stauffer RL, Kardos NL, Hedges SB: Molecular evidence for the early colonization of land by fungi and plants. Science 200I, 293: I I29-II33.

91. Joint Genome Institute [http://genome.jgi-psf.org/Poptr I I/ Poptrl I.home.html]

92. Thompson JD, Higgins DG, Gibson TJ: Clustal W - Improving the sensitivity of progressive multiple sequence alignment through sequence weighting, position-specific gap penalties and weight matrix choice. Nucleic Acids Res 1994, 22:4673-4680.

93. Page RDM: TREEVIEW: An application to display phylogenetic trees on personal computers. CABIOS 1996, I 2:357-358.

94. Sali A, Blundell TL: Comparative protein modeling by satisfaction of spatial restraints. J Mol Biol 1993, 234:779-8I5.

95. MODELLER [http://www.salilab.org/modeller/]

96. Canutescu AA, Shelenkov AA, RL Dunbrack Jr: A graph-theory algorithm for rapid protein side-chain prediction. Protein Sci 2003, I 2:2001-2014.

97. Lindahl E, Hess B, Spoel D van der: GROMACS 3.0: a package for molecular simulation and trajectory analysis. J Mol Modeling 200I, 7:306-3I7.

98. GROMACS [http://www.gromacs.org/]

99. Laskowski RA, MacArthur MW, Moss DS, Thornton JM: PROCHECK-A program to check the stereochemical quality of protein structures. J Appl Cryst I993, 26(Part 2):283-29I.

100. Smart OS, Neduvelil JG, Wang X, Wallace BA, Sansom MSP: HOLE: A program for the analysis of the pore dimensions of ion channel structural models. J Mol Graphics 1996, I4:354-360.

10I. Gene Expression Omnibus [http://www.ncbi.nlm.nih.gov/geo/]

102. NetAffx ${ }^{\mathrm{TM}}$ Analysis Center [https://www.affymetrix.com/analy sis/index.affx]

103. Poplar eFP Browser [http://bar.utoronto.ca/efppop/cgi-bin/efp Web.cgi]

Publish with BioMed Central and every scientist can read your work free of charge

"BioMed Central will be the most significant development for disseminating the results of biomedical research in our lifetime. "

Sir Paul Nurse, Cancer Research UK

Your research papers will be:

- available free of charge to the entire biomedical community

- peer reviewed and published immediately upon acceptance

- cited in PubMed and archived on PubMed Central

- yours - you keep the copyright
BioMedcentral 